Full length article

\title{
Injectable and tunable hyaluronic acid hydrogels releasing chemotactic and angiogenic growth factors for endodontic regeneration
}

\author{
Cristiana R. Silva ${ }^{\mathrm{a}, \mathrm{b}}$, Pedro S. Babo ${ }^{\mathrm{a}, \mathrm{b}}$, Maurizio Gulino ${ }^{\mathrm{a}, \mathrm{b}}$, Lígia Costa ${ }^{\mathrm{a}, \mathrm{b}}$, Joaquim M. Oliveira ${ }^{\mathrm{a}, \mathrm{b}, \mathrm{c}}$, \\ Joana Silva-Correia ${ }^{\mathrm{a}, \mathrm{b}}$, Rui M.A. Domingues ${ }^{\mathrm{a}, \mathrm{b}, \mathrm{c}}$, Rui L. Reis ${ }^{\mathrm{a}, \mathrm{b}, \mathrm{c}}$, Manuela E. Gomes ${ }^{\mathrm{a}, \mathrm{b}, \mathrm{c}, *}$ \\ a 3B's Research Group, I3Bs - Research Institute on Biomaterials, Biodegradables and Biomimetics, University of Minho, Headquarters of the European Institute of Excellence on \\ Tissue Engineering and Regenerative Medicine, AvePark, Parque de Ciência e Tecnologia, Zona Industrial da Gandra, 4805-017 Barco, Guimarães, Portugal \\ b ICVS/3B's - PT Government Associate Laboratory, Braga/Guimarães 4805-017, Portugal \\ ${ }^{\mathrm{c}}$ The Discoveries Centre for Regenerative and Precision Medicine, Headquarters at University of Minho, Avepark, 4805-017 Barco, Guimarães, Portugal
}

\section{A R T I C L E I N F O}

\section{Article history:}

Received 15 March 2018

Received in revised form 13 July 2018

Accepted 17 July 2018

Available online 18 July 2018

\section{Keywords:}

Injectable hydrogel

Growth factors-controlled release

Dental pulp cells

Neo-angiogenesis

Migration

Endodontic tissue regeneration

\begin{abstract}
A B S T R A C T
Bioengineered soft tissues on any meaningful scale or complexity must incorporate aspects of the functional tissue, namely a vasculature, providing cells oxygen and nutrients critical for their survival. However, the ability of tissue engineering strategies to promote a fast revascularization is critically limited. Particularly in endodontic regenerative therapies, the complicated anatomy of the root canal system, and the narrow apical access limit the supply of new blood vessels and pulp tissue ingrowth.

Here we characterize the viscoelastic and microstructural properties of a class of injectable hyaluronic acid (HA) hydrogels formed in situ, reinforced with cellulose nanocrystals (CNCs) and enriched with platelet lysate (PL), and test its ability to promote cells recruitment and proangiogenic activity in vitro. The incorporation of CNCs enhanced the stability of the materials against hydrolytic and enzymatic degradation. Moreover, the release of the chemotactic and pro-angiogenic growth factors (GFs) (PDGF and VEGF) from the PL-laden hydrogels showed an improved sustained profile proportional to the amount of incorporated CNCs. The PL-laden hydrogels exhibited preferential supportive properties of encapsulated human dental pulp cells (hDPCs) in in vitro culture conditions.

Finally, PL-laden hydrogels stimulated chemotactic and pro-angiogenic activity by promoting hDPCs recruitment and cell sprouting in hDPCs/human umbilical vein endothelial cell co-cultures in vitro, and in an ex vivo model. These results support the use of the combined system as a scaffold for GFs delivery and cells recruitment, thereby exhibiting great clinical potential in treating injuries in vascularized tissues.
\end{abstract}

\section{Statement of Significance}

Innovative strategies for improved chemotactic and pro-angiogenic features of TE constructs are needed. In this study, we developed an injectable $\mathrm{HA} / \mathrm{CNC} / \mathrm{PL}$ hydrogel with improved structural and biologic properties, that not only provide a sustained release of chemotactic and proangiogenic GFs from PL but also enhance the cells' viability and angiogenic activity. As a result of their unique traits, the developed hydrogels are ideally suited to simultaneously act as a GFs controlled delivery system and as a supportive matrix for cell culture, recruitment, and revascularization induction, holding great potential for the regeneration of vascularized soft tissues, such as the dentin-pulp complex.

(c) 2018 Acta Materialia Inc. Published by Elsevier Ltd. All rights reserved.

\section{Introduction}

Host-derived vascularization of implanted 3D structures, is largely limited by the overall difficulty of host cells to invade and

\footnotetext{
* Corresponding author.

E-mail address: megomes@dep.uminho.pt (M.E. Gomes).
}

form functional capillaries [1]. This limits the therapeutic potential of implanted constructs due to lack of nutrient delivery and waste removal, which result in the formation of a necrotic core [2], while impair the tissue integration of the grafted constructs. The rapid induction of angiogenesis represents the major challenge for endodontic regenerative therapies, in the early stages of dentinpulp regeneration [3]. The complicated anatomy of the root canal 
system, and the narrow apical access limit the blood supply and pulp tissue ingrowth. Thus, appropriate engineered constructs are critically needed to ensure the rapid vascularization upon implantation, and, therefore, the sustained diffusion of nutrients, oxygen and metabolites [4], the income of host cells and/or the survival of transplanted cells.

Biologically relevant hydrogels can address this functional feature through microfabrication of vessel-like structures, or biological formation of a vascular system as a result of cell-cell interactions and their cross-communication with growth factors (GFs) and the extracellular matrix (ECM) [4]. Hydrogels are crosslinked hydrophilic polymer networks, presenting physical characteristics similar to soft tissues, such as the dental pulp tissue. Due to hydrogels high water content (usually $\geq 90 \%$ ), they provide stable and flexible wet matrices with adequate porosity for diffusion of nutrients, and cellular waste [5,6]. Hydrogels can also efficiently incorporate and release biological agents, important to enhance the revascularization of the root canal system, and the simultaneous regeneration of the dentin-pulp complex [7]. The injectable hydrogels are of particular interest as they can be delivered through minimally invasive surgical procedures [8-11], and produced in situ to conform to the required defect dimensions [12]. Their ability to be easily dispersed inside any closed complex space, has propelled studies in terms of its potential application in tissue engineering (TE) approaches with the same complexity of endodontic regenerative therapies.

Building on a previous study [13], here we propose the amelioration of a class of in situ injectable hyaluronic acid (HA) hydrogels based on hydrazone crosslinking between hydrazide and aldehyde groups, reinforced with cellulose nanocrystals (CNCs), and enriched with human platelet lysate (PL). Injectable HA-based hydrogels compose a promising approach for endodontic regenerative therapies due to HA structural and physiological functions within ECM [14], enhancing the biocompatibility and biological recognition, while maintaining the homeostatic integrity of tissues [15]. Based on their chemistry, these hydrogels can be chemically cross-linked, and functionalized with physical or biochemical cues to improve their mechanical strength or promote angiogenesis and/or stem cell migration, and enhance pulp-dentin regeneration [16]. CNCs have shown to significantly enhance the stability and mechanical performance of HA hydrogels [13,17]. Moreover, the higher density of negative charged sulfate groups presented in CNCs' surface may play a significant role on GFs immobilization and release profiles, due to their ability to potentially act as sulfated glycosaminoglycan, which are known to noncovalently bind and modulate cytokines, GFs, and other ECM proteins $[18,19]$. PL is rich in chemotactic and proangiogenic GFs (platelet-derived growth factor (PDGF), vascular endothelial growth factor (VEGF)) [20], which when incorporated and released from HA-derived hydrogels is expected to stimulate the migration and proliferation of encapsulated cells and/or endogenous cells, and induce the revascularization of the root canal system and simultaneous pulp tissue ingrowth.

As a proof-of-concept and to test these hypotheses, we incorporated increasing concentrations of CNCs, and characterized their morphological and biomechanical/viscoelastic properties. The in vitro biodegradation of the hydrogels was studied by monitoring the release of GFs from the polymeric matrix, and by monitoring the weight loss and swelling properties. The biological performance of selected cell/hydrogel was evaluated towards human dental pulp cells (hDPCs) in vitro. Additionally, we tested the ability of the PL-laden hydrogels to recruit dental pulp-origin cells, and promote new vessel-like structures sprouting in vitro and in an ex vivo model, as a critical prerequisite for dentin-pulp regeneration.

\section{Materials and methods}

\subsection{Production of hydrogels precursors}

CNCs, previously isolated from microcrystalline cellulose (MCC) by sulfuric hydrolysis [13,21]' were functionalized by sodium periodate $\left(\mathrm{NaIO}_{4}\right)$ oxidation, following the procedure described elsewhere with slight modifications [22,23].

Hydrazide-modified HA (ADH-HA) was produced according to a well-established carbodiimide chemistry [24-26], whereas aldehyde-modified HA (a-HA) was synthesized following a siteselective $\mathrm{NaIO}_{4}$ oxidation, as described elsewhere, with minor adaptations [27].

Moreover, PL was collected and processed from different platelet concentrates, by repeated freezing and melting cycles, as previously described [28].

The experimental procedures and characterizations of hydrogels' precursors are documented in detail in supporting information.

\subsection{Development of PL-laden hydrogels}

HA hydrogels incorporating CNCs and enriched with PL were produced through hydrazone cross-linking chemistry between hydrazide/amine and aldehyde groups (Fig. 1), by adaptation of a procedure developed elsewhere [13]. The hydrogels were prepared at room temperature by mixing equal amounts of aldehyde and hydrazide derivatives of HA. A double-barrel syringe, fitted with a static mixer placed at the outlet, all from Medmix (Germany) was used to produce the injectable hydrogels. For HA/CNCs hydrogels without PL, barrel A was filled only with ADH-HA, previously dissolved in Dulbecco's phosphate buffered saline (DPBS, Life Technologies, USA) ( $2 \% \mathrm{w} / \mathrm{v}$ solution) and barrel $\mathrm{B}$ with a$\mathrm{HA}(2 \% \mathrm{w} / \mathrm{v})$ and a-CNCs dispersions at $0.25,0.5,0.75$, and $1 \mathrm{wt}$ $\%$ concentration. For PL-laden hydrogels, barrel A was filled with ADH-HA previously dissolved in PL solution, and barrel $B$ had the same precursors as barrel B for hydrogels without PL. Before extrusion, the viscous ADH-HA solution was briefly centrifuged ( 1 pulses of $10 \mathrm{~s}$ ) to remove any remaining bubble, capable of negatively interfere with the polymerization process. The hydrogel precursor solutions were then hand extruded into cylindrical acrylate molds ( $5 \mathrm{~mm}$ in diameter $\times 5 \mathrm{~mm}$ in height). The final PL-laden hydrogels were composed of $1 \mathrm{wt} \%$ ADH-HA, $1 \mathrm{wt} \%$ a-HA, and 0.125 to $0.5 \mathrm{wt} \%$ a-CNCs in $50 \mathrm{v} / \mathrm{v} \%$ PL solution. Table 1 summarizes the different conditions used to develop the hydrogels, as well the abbreviations used throughout the manuscript.

\subsection{In situ gelation time}

The apparent in situ gelation time of the hydrogels was measured at room temperature as previously described [25]. A magnetic stirring bar was placed in the center of a $100 \mu \mathrm{L}$ droplet of ADH-HA aqueous solution (2 wt\%) in saline or PL, in a Petri dish and stirred at $160 \mathrm{rpm}$ using a magnetic stirrer. $100 \mu \mathrm{L}$ of a-HA or a-HA/a-CNCs solutions ( $2 \mathrm{wt} \%$ a-HA, 0, 0.5, and $1 \mathrm{wt} \%$ a-CNCs) was then added to the ADH-HA drop while stirring. The gelation time was counted from the mixing point to the gel state, determined when the solution formed a solid globule after a certain reaction period, which was defined as the apparent gelation time. The assay was performed in triplicate for each condition. All the process was observed in real time using a stereo microscope (Stemi 1000, Zeiss) with a lamp (Schott KL 200, Germany), and recorded using a digital camera (Canon G12, Japan). 


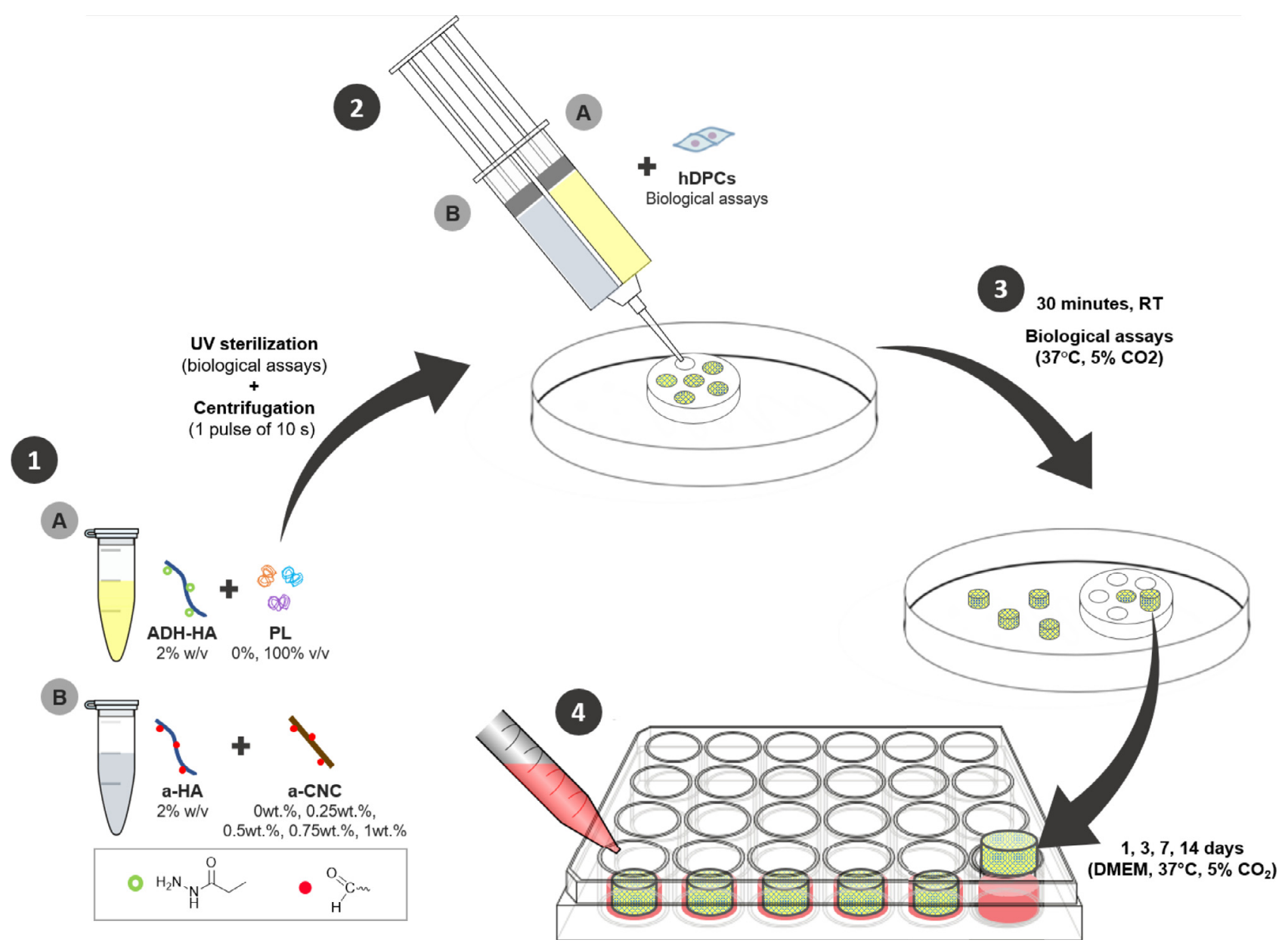

Fig. 1. Schematic representation of the proposed in situ crosslinking system. (1) Preparation of the (A) hydrazide-functionalized hyaluronic acid (ADH-HA) solution with or without PL and (B) aldehyde functionalized hyaluronic acid (a-HA), with or without aldehyde-modified CNCs (a-CNCs). (2) Preparation of the HA-based hydrogels through the co-injection of the polymeric solutions, using a double barrel syringe. For biological assays human dental pulp cells (hDPCs) were added to solution (A) before injection in the molds. (3) Stable hydrogels formed upon cross-link reaction between the hydrazide/amine and carbonyl moieties of precursor components. (4) Incubation of the prepared hydrogels in physiological conditions, up to 14 days, to assess their biological properties towards hDPCs.

Table 1

Summary of conditions used for the development of injectable HA/CNCs hydrogels.

\begin{tabular}{llll}
\hline $\begin{array}{l}\text { Formulation } \\
\text { designation }\end{array}$ & $\begin{array}{l}\text { Concentration of } \\
\text { a-HA/ADH-HA (wt\%) }\end{array}$ & $\begin{array}{l}\text { Concentration of } \\
\mathrm{a}-\mathrm{CNCs}(\mathrm{wt} \%)\end{array}$ & $\begin{array}{l}\text { Concentration of } \\
\mathrm{PL}(\mathrm{v} / \mathrm{v} \%)\end{array}$ \\
\hline $0 \% \mathrm{CNC}$ & & 0 & \\
$0.125 \% \mathrm{CNC}$ & & 0.125 & - \\
$0.25 \% \mathrm{CNC}$ & $1 / 1$ & 0.25 & \\
$0.375 \% \mathrm{CNC}$ & & 0.375 & \\
$0.5 \% \mathrm{CNC}$ & & 0.5 & \\
$0 \% \mathrm{CNC} / \mathrm{PL}$ & & 0 & 50 \\
$0.125 \% \mathrm{CNC} / \mathrm{PL}$ & & 0.125 & \\
$0.25 \% \mathrm{CNC} / \mathrm{PL}$ & $1 / 1$ & 0.25 & \\
$0.375 \% \mathrm{CNC} / \mathrm{PL}$ & & 0.375 & \\
$0.5 \% \mathrm{CNC} / \mathrm{PL}$ & & 0.5 & \\
\hline
\end{tabular}

\subsection{Hydrogel microstructure}

Cryo-Scanning Electron Microscopy (Cryo-SEM) analysis was performed to assess the hydrogels' structure, using a High Resolution Scanning Electron Microscope with X-Ray Microanalysis and Cryo-SEM experimental facilities (JEOL JSM 6301F/ Oxford INCA Energy 350/Gatan Alto 2500). The specimens were rapidly cooled by plunging it into sub-cooled nitrogen (slush nitrogen), and transferred under vacuum to the cold stage of the preparation chamber. Each sample was fractured, exposing their inner structures, sublimated ('etched') for $120 \mathrm{~s}$ at $-90^{\circ} \mathrm{C}$, and coated with $\mathrm{Au} / \mathrm{Pd}$ by sputtering for $40 \mathrm{~s}$ and with a $12 \mathrm{~mA}$ current. The sample was then transferred into the SEM chamber, and imaged at $-150^{\circ} \mathrm{C}$. The measurement of pores' diameter of each sample was performed using ImageJ software. At least 100 pores for each formulation were randomly selected from different SEM images.

\subsection{Assessment of the mechanical/viscoelastic properties of hydrogels}

The dynamic viscoelastic behavior of developed hydrogels as function of time was investigated by using a Kinexus Prot Rheometer from Malvern (UK), and a $20 \mathrm{~mm}$ diameter parallel plate geometry. Samples were carefully injected in the middle of the base plate, and covered with oil to avoid dehydration. The experiments were carried out at $37^{\circ} \mathrm{C}$, under constant strain amplitude (1\%) and frequency $(1 \mathrm{~Hz})$, for a period of $90 \mathrm{~min}$. The strain used had been found to be within the linear viscoelastic region for all hydrogels. At least four different samples were tested for each composition with the same experimental settings.

\subsection{Enzymatic degradation and swelling assays}

The enzymatic degradation behaviour of the hydrogels was tested in simulated physiological conditions to conclude about their biodegradability and stability, according to their CNCs 
content. In this sense, the prepared hydrogels $(n=4)$ were initially weighed (Wi) and subsequently incubated in $1.2 \mathrm{~mL}$ PBS buffer $\mathrm{pH}$ 7.4 containing $2.8 \mathrm{U} / \mathrm{mL}$ hyaluronidase (HAase) from bovine testes, Type IV-S (Sigma-Aldrich, USA) at $37^{\circ} \mathrm{C}$, under constant agitation in a horizontal orbital shaker at $180 \mathrm{rpm}$. At each preselected time point ( $0 \mathrm{~h}, 3 \mathrm{~h}, 8 \mathrm{~h}, 22 \mathrm{~h}$ and 2, 4, 5, 6, 8, 11 and 12 days), the hydrogels were weighed (Wf), and the incubation medium was stored and replaced for fresh HAase solution. Measurements were performed until complete degradation of the samples and the time at which the gel disappeared was recorded. The degradation was calculated using the following equation: hydrogel mass $(\%)=W_{\mathrm{f}}$ $\mathrm{W}_{\mathrm{i}} \times 100 \%$.

The swelling properties of the hydrogels were also analyzed by assessing their percentage swelling ratio. The prepared hydrogels $(\mathrm{n}=4)$ were initially weighed $(\mathrm{Wi})$, immersed in PBS $(\mathrm{pH} 7.4)$, and kept at $37^{\circ} \mathrm{C}$ for 20 days. At different time points $(0 \mathrm{~h}, 3 \mathrm{~h}, 8$ $\mathrm{h}$ and $22 \mathrm{~h}$ and $2,3,6,7,11,15$ and 20 days), the immersed hydrogels were removed, the excess of PBS was absorbed with a filter paper, and immediately weighed (Wf). Percentage swelling ratio was expressed as the percentage ratio between the weight of swollen gel and fresh gel. It could be derived from: swelling ratio (\%) = $\mathrm{W}_{\mathrm{f}}-\mathrm{W}_{\mathrm{i}} / \mathrm{W}_{\mathrm{i}} \times 100 \%$.

\subsection{Release of pro-angiogenic platelet lysate-origin growth factors}

The ability of the developed PL-based hydrogels to release PDGF-BB and VEGF was assessed by enzyme-linked immunosorbent assay (ELISA). The content of these GFs was assessed in supernatant taken from the incubation solutions, withdrawn and frozen at $-20{ }^{\circ} \mathrm{C}$ at pre-selected time points, during enzymatic degradation assays of the PL-based hydrogels. The quantitative measurement of the mentioned GFs was performed using two similar kits, Human PDGF-BB ELISA Development Kit and Human vEGF ELISA Development Kit (Peprotech, USA), and according to manufacturer's specifications. The optical density was monitored with multiwell microplate reader (Synergy HT, Bio-Tek Instruments, USA) at $405 \mathrm{~nm}$ with wavelength correction set at $650 \mathrm{~nm}$. Each reading was performed in duplicate for four samples of each formulation. The cumulative release of each GF was calculated as follows:

cumulative release $(\%)=\mathrm{M}_{\mathrm{t}} / \mathrm{M}_{0} \times 100 \%$,

where $\mathrm{M}_{0}$ is the amount of VEGF or PDGF preloaded into hydrogel and Mt is the amount of VEGF or PDGF released from the PLloaded hydrogel at time $t$.

The ability of the HA/PL hydrogels to retain GFs, namely the entrapment in the hydrogel matrix and the stability over time of PDGF-BB and VEGF was assessed by Western Blot. For this purpose, PL-laden hydrogels were incubated in basal medium for $0,7,14$ and 21 days at $37^{\circ} \mathrm{C}$. After each incubation time, hydrogels were washed twice in PBS, and frozen at $-80^{\circ} \mathrm{C}$ inside $1.5 \mathrm{~mL}$ eppendorf tubes. Before analysis, HA/PL hydrogels were thawed at room temperature, and $1 \mathrm{~mL}$ of extraction buffer $(150 \mathrm{mM} \mathrm{NaCl}$ (Panreac Quimica, Spain), $100 \mathrm{mM}$ Tris- $\mathrm{HCl}, 2 \mathrm{mM}$ ethylenediaminetetraacetic acid, 1\% Triton X-100 (Sigma-Aldrich, Germany), 0.5\% sodium deoxycholate, and 1:100 protease inhibitor cocktail, all from Sigma-Aldrich (USA)) was added to each hydrogel. After milling in an ultrasonic processor (VCX-130 PB-220, Sonics, USA) the hydrogels' slime was incubated for $1 \mathrm{~h}$ at $4{ }^{\circ} \mathrm{C}$, under constant agitation in a horizontal orbital shaker (SC2D000580, SK-O330PRO, SCANSCI). Thereafter, the samples were centrifuged for 20 min at $13,500 \mathrm{~g}$, and the supernatant was replaced by $30 \mu \mathrm{L}$ of $0.9 \% \mathrm{w} / \mathrm{v} \mathrm{NaCl}$ solution, and $10 \mu \mathrm{L}$ of loading buffer ( $4 \mathrm{w} / \mathrm{v} \% \mathrm{SDS}$ (NZYTech, Portugal), $10 \mathrm{v} / \mathrm{v} \%$ 2-mercaptoethanol, $20 \mathrm{v} / \mathrm{v} \%$ glycerol, $0.004 \mathrm{w} / \mathrm{v} \%$ bromophenol blue, $0.125 \mathrm{M}$ Tris- $\mathrm{HCl} \mathrm{pH} 6.8$, all from Sigma-Aldrich (USA)). Subsequently, the samples were heated at $95^{\circ} \mathrm{C}$ for $5 \mathrm{~min}$, and centrifuged at $13,500 \mathrm{~g}$ in a gyrospin centrifuge (ScanSpeed Mini, Labogene, Denmark) for $1 \mathrm{~min}$.

The proteins present in the hydrogel macerate were separated by electrophoresis in a $12 \%$ resolving and $4 \%$ stacking SDS-PAGE gel (Fluka, USA). A 10-250 kDa molecular weight marker (PageRuler Plus Prestained Protein Ladder, Thermo Fisher Scientific, USA) was used as reference. Following electrophoretic separation, the proteins were transferred from the SDS-PAGE onto nitrocellulose membranes (GE Healthcare, UK), using routine wet stage transfer protocols.

Afterwards, the membranes were blocked in $3 \%$ bovine serum albumin (BSA, Sigma-Aldrich, USA) in tris-buffered saline with Tween 20 (TBST) buffer (20 mM tris base (Nzytech, Portugal), $150 \mathrm{mM} \mathrm{NaCl}, 0.1$ v/v\% Tween 20 (Sigma-Aldrich, USA), pH 7.5) at room temperature for $1 \mathrm{~h}$. Then, the membranes were incubated overnight in the primary antibody solution against the target protein at $4{ }^{\circ} \mathrm{C}$. To detect PDGF-BB, membrane was incubated with anti-mouse PDGF-BB (ab51869, Abcam, UK) 1:200 in blocking buffer. The antibody anti-mouse VEGF-A (ab171828, Abcam, UK) was diluted in TBST buffer $(1: 1000)$, and used to detect VEGF. Then, the blot was rinsed three times for 5 min with TBST buffer, and incubated in the alkaline phosphatase horse anti-mouse IgG secondary antibody (AP-2000, Vector Laboratories, USA), diluted in blocking buffer solution (1:500), for $1 \mathrm{~h}$ at room temperature. For signal development, a colorimetric alkaline phosphatase conjugate substrate (Bio-Rad, USA) was applied to the blot, according to the manufacturer's recommendation. Lastly, the blot was rinsed three times for 5 min with TBST buffer, followed by imaging and data analysis. This assay was performed in duplicate.

\subsection{Expansion of human dental pulp cells (hDPCs)}

Human dental pulp cells (hDPCs) were isolated from human third molars under the scope of a previous study [29]. These cells were positive for mesenchymal stem cell markers such as CD73, CD90, CD105 and CD44 with over 98\% of cells expressing these markers, and negative for the hematopoietic markers CD34 and CD45 (<1\%) [29]. hDPCs were cultured in $75 \mathrm{~cm}^{3}$ flasks with Dulbecco's Modified Eagle's Medium - low glucose (DMEM, SigmaAldrich, USA) and supplemented with $10 \%$ fetal bovine serum (FBS, Life Technologies, USA), and $1 \%$ antibiotic/antimycotic (Life Technologies, USA). The culture medium was replaced every 2 days, and maintained semiconfluent. All cultures were incubated at $37^{\circ} \mathrm{C}$ in a $5 \% \mathrm{CO}_{2}$ high-humidity environment. hDPCs at passage 6 were used in these studies.

\subsection{Expansion of human umbilical vein endothelial cells (HUVECS)}

Human umbilical vein endothelial cells (HUVECs, Lonza, USA), were firstly expanded in $75 \mathrm{~cm}^{2}$ flasks previously coated with $0.7 \%$ gelatin (gelatin from porcine skin, Type A, Sigma-Aldrich, USA) solution. Before cells being cultured, the flask was washed with DPBS to remove the excess of gelatin. Cells were cultured with endothelial cell basal media (Millipore S.A.S, France) supplemented with $5 \mathrm{ng} / \mathrm{mL}$ rh VEGF, $5 \mathrm{ng} / \mathrm{mL}$ rh epidermal growth factor (EGF), $5 \mathrm{ng} / \mathrm{mL}$ rh basic fibroblast growth factor (FGF), $15 \mathrm{ng} / \mathrm{mL}$ rh insulin-like growth factor-1 (IGF-1), $50 \mu \mathrm{g} / \mathrm{mL}$ ascorbic acid, 1.0 $\mu \mathrm{g} / \mathrm{mL}$ hydrocortisone hemisuccinate, $0.75 \mathrm{U} / \mathrm{mL}$ heparin sulfate, $10 \mathrm{mM}$ L-glutamine, and 2\% of FBS, all from Millipore S.A.S (France), until reaching an adequate number. Medium was replaced every 2 days, and the culture was maintained semiconfluent. All cultures were incubated at $37{ }^{\circ} \mathrm{C}$ in a $5 \% \mathrm{CO}_{2}$ high-humidity environment. HUVECs at passage 5 were used in this study. 


\subsection{Encapsulation of hDPCs}

The production of the hydrogels was conducted in similar conditions as described in Section 2.2, but under sterile conditions (Fig. 1). Briefly, a-HA was dissolved in $1 \times$ DPBS containing 0.5 and $1 \mathrm{wt} \%$ a-CNCs, and ADH-HA was dissolved separately in $1 \mathrm{X}$ DPBS or PL solution, at a concentration of $2 \mathrm{wt} \%$ overnight at room temperature. All solutions were sterilized by UV irradiation (254 $\mathrm{nm}$ ) for $120 \mathrm{~min}$ prior to cell encapsulation. Cultured cells were detached by trypsin and centrifuged at $300 \mathrm{~g}$ for $5 \mathrm{~min}, 22^{\circ} \mathrm{C}$. The obtained cell pellet was resuspended in culture media, counted using a hemocytometer and finally centrifuged. The supernatant was discarded and $8 \times 10^{6}$ of hDPCs per $\mathrm{mL}$ were resuspended in the ADH-HA or ADH-HA/PL solutions. The cell suspension was thoroughly mixed to homogenize the distribution of the cells within the matrix. The cells mixture was added to the barrel A of the double-barrel syringe and the barrel B was filled with a-HA and a-CNCs. The hydrogel precursor solutions were then carefully hand extruded into cylindrical Teflon molds $(5 \mathrm{~mm}$ in diameter $\times 5 \mathrm{~mm}$ in height), and incubated at $37^{\circ} \mathrm{C}$ for $30 \mathrm{~min}$ to form a solid gel. The final hydrogels' composition was as indicated in Table 1 , but incorporating a cell cargo of $4 \times 10^{6} \mathrm{hDPCs} / \mathrm{mL}$. The hydrogels with encapsulated cells were cultured in 24-well plates in basal media for $1,3,7$, and 14 days $\left(37^{\circ} \mathrm{C}, 5 \% \mathrm{CO}_{2}\right)$ with the replacement of the culture media twice a week.

\subsection{Hydrogel cytotoxicity over encapsulated hDPCS}

The metabolic activity of the encapsulated hDPCs was quantitatively assessed by Alamar Blue fluorescent assay, according to the manufacturer's instructions [30]. At each pre-settled time point, samples were rinsed twice with PBS and transferred to new nonadherent 24-well plates. Each sample was incubated in $10 \% \mathrm{v} / \mathrm{v}$ Alamar Blue (Bio-Rad, England) in warm culture medium for $4 \mathrm{~h}$ at $37^{\circ} \mathrm{C}$, in standard culture conditions. Then, $50 \mu \mathrm{L}$ of supernatant were transferred to transparent 96-well plates. Four hydrogels of each condition were analysed, and the fluorescence readings were performed in triplicate. The fluorescence was measured using a Synergy HT microplate reader (Bio-Tek Instruments, USA) at excitation and emission wavelength of 530 and $590 \mathrm{~nm}$, respectively. After the fluorescence readings, the samples were washed with PBS, transferred to eppendorf tubes containing $1 \mathrm{~mL}$ of sterile ultrapure water and stored at $-80{ }^{\circ} \mathrm{C}$ for further DNA extraction and quantification.

Cell proliferation was evaluated by quantifying the total amount of double-stranded DNA present at different culture times. DNA quantification was performed using the Quant-IT ${ }^{\mathrm{TM}}$ PicoGreen dsDNA Assay Kit (Molecular Probes, Invitrogen, USA), and according to the manufacturer's instructions. Before the analysis, the samples were thawed at room temperature and sonicated for 3 cycles of $5 \mathrm{~s}$. The fluorescence was measured at an excitation wavelength of $485 / 20 \mathrm{~nm}$ and at an emission wavelength of $528 / 20 \mathrm{~nm}$. Four hydrogels of each condition were analysed, and the fluorescence readings were performed in triplicate for each sample.

The cell morphology was assessed and studied by staining the nucleus and actin filaments of the hDPCs after 14 days of culture, to study the ability of developed hydrogels to support the cell adhesion. Samples were washed 3 times with PBS, before and after fixation with $10 \%(\mathrm{v} / \mathrm{v})$ neutral buffered formalin (ThermoFisher Scientific, USA) for $30 \mathrm{~min}$ at room temperature. Then, samples were incubated in $0.2 \%(\mathrm{v} / \mathrm{v})$ Triton X-100 (Sigma-Aldrich, Germany) prepared in PBS, for $1 \mathrm{~h}$ at room temperature and under constant agitation. After washed with PBS, fixed samples were incubated in $1 \mathrm{~mL}$ of the PBS solution containing 4,6-diamidino2-phenyindole dilactate (DAPI, Biotium, USA) 1:1500 v/v, and
Phalloidin (Phalloidin-Tetramethylrhodamine B isothiocyanate from Amanita phalloides Sigma-Aldrich, USA) 1:500 v/v, for $1 \mathrm{~h}$ at room temperature, under agitation. After rinsing in PBS to reduce background fluorescence, hydrogels were mounted on microscopic slides and visualized under a confocal microscope (Leica TCS SP8, Microsystems, Wetzlar, Germany), and representative micrographs were taken. Each experiment was performed in duplicate.

\subsection{In vitro evaluation of the chemotactic and proangiogenic properties of the HA/PL hydrogels}

The ability of the proposed system to recruit progenitor cells and promote neovascularization was assessed in in vitro conditions, by analysing the sprouting of encapsulated pure hDPCs pellets and co-cultures of hDPCs and HUVECs. Briefly, cultured cells were detached by trypsin and, centrifuged at $300 \mathrm{~g}$ for $5 \mathrm{~min}$, at $22^{\circ} \mathrm{C}$. After counting, HUVECs were stained for $20 \mathrm{~min}$ with green CellTrace CFSE dye (C34554, Molecular Probes, Invitrogen, USA) in dimethylsulfoxide (DMSO, Molecular Probes, Invitrogen, USA) diluted in PBS 1:1000 v/v, whereas hDPCs were stained in red with CellTracker CM-Dil dye in DMSO (C7000, Molecular Probes, Invitrogen, USA) diluted in PBS 1:500 v/v. A culture medium volume equivalent to five times the original staining solution was added to each cell type, and incubated for more 5 min before centrifugation. Then, HUVECs were mixed with hDPCs at a ratio of $1: 1$ (total of $2 \times 10^{5}$ cells), and pelleted by two successive centrifugation cycles at $2864 \mathrm{~g}$ for $5 \mathrm{~min}, 20^{\circ} \mathrm{C}$ and, incubated overnight. Likewise, pure hDPCs pellets containing $2 \times 10^{5}$ cells were produced as controls. Cell pellets were encapsulated into hydrogels by injecting the polymeric solutions over the pellets placed in the bottom of the acrylate moulds ( $5 \mathrm{~mm}$ high $\times 5 \mathrm{~mm}$ diameter), and let to polymerize for $30 \mathrm{~min}\left(37^{\circ} \mathrm{C}, 5 \% \mathrm{CO}_{2}\right)$. Hydrogels were then removed along with the molds ( $5 \mathrm{~mm}$ in diameter $\times 5 \mathrm{~mm}$ in height), placed inside a transwell (VWR, USA), mimicking the natural nutrition provision of root canal system, and incubated in DMEM medium up to 3 days. The sprouting was assessed over time by fluorescence inverted microscope (Axio Observer, Zeiss, Germany). The measurement of the sprouting length of each sample was performed using Image J software. For each fluorescence image, the pellet was divided into four quadrants and two sprouts were randomly selected from each quadrant and measured. Hydrogels were produced in triplicate for each composition.

\subsubsection{Immunocytochemistry}

At the end of the 3rd day in culture, hydrogels were rinsed 3 times with PBS, before and after fixation with $10 \%(\mathrm{v} / \mathrm{v})$ neutral buffered formalin for $30 \mathrm{~min}$ at room temperature. Before immunostaining, samples were permeabilized for $1 \mathrm{~h}$ with $0.2 \%$ (v/v) Triton X-100 (Sigma-Aldrich, Germany) prepared in PBS, at room temperature and under constant agitation. Then, samples were washed 3 times with PBS to remove remaining residues and the cells nuclei was counterstained with DAPI 1:1500 v/v for $1 \mathrm{~h}$ at room temperature, under agitation. After rinsing in PBS to reduce background fluorescence, hDPCs pellets were immunostained using primary antibodies against CD90 (mouse antihuman CD90 APC, Pharmingen, USA, \#559869, 1:100), and alexafluor 594 donkey anti-mouse (A21203, Invitrogen, USA, 1:1000) was used as secondary antibody. The coculture pellets were immuno-stained against CD106 (mouse anti-human CD106 (VCAM-1) PE, Pharmingen, USA, 555647, 1:100) and alexafluor 488 rabbit anti-mouse (A11059, Invitrogen, USA, 1:1000) was used as secondary antibody, followed by immune-staining of the mesenchymal cells present in the co-cultures with CD90 (mouse anti-human CD90 APC, Pharmingen, USA, \#559869, 1:100). Samples were incubated with the primary antibodies at $4{ }^{\circ} \mathrm{C}$ overnight. 
After incubation with primary antibodies, all samples were washed 3 times with PBS for 15 min. Then, samples were incubated with fluorescent-labelled secondary antibodies at room temperature for $1 \mathrm{~h}$, prevented from light exposure. All antibodies were diluted in antibody dilution buffer (Dako, USA). After washed 3 times, immunolabeled samples were analysed by confocal laser microscopy (Leica TCS SP8, Microsystems, Wetzlar, Germany).

\subsection{Ex vivo evaluation of the HA/PL hydrogels ability to promote neo- vascularization}

\subsubsection{Chick chorioallantoic membrane assay}

Chick chorioallantoic membrane (CAM) assay was conducted to evaluate the effectiveness of the PL-laden hydrogels to promote neo-vascularization, according to an optimized adaptation described by Silva-Correia et al. [31]. White fertilized chicken eggs ( $n=120$; Pintobar, Portugal) were incubated at $37^{\circ} \mathrm{C}$ for 3 days (Laboratory Incubator model B8420; Termaks). Two small holes were then created in the longitudinal extremities of the egg to promote CAM dissociation from the egg shell membrane. A circular window was also created in the egg shell to assess CAM and embryo viability. The opening in the egg shell was then sealed with transparent tape $(\sim 50 \times 30 \mathrm{~mm})$ to avoid dehydration and the eggs were incubated again at $37^{\circ} \mathrm{C}$ until day 10 of embryonic development. At day 10, the different sterile HA-based formulations discs (1:1 HA/ADH-HA wt\%; $4 \mathrm{~mm}$ in diameter) denoted by $0 \% \mathrm{CNC}, 0 \% \mathrm{CNC} \mathrm{w} / \mathrm{hDPCs}\left(4 \times 10^{6}\right.$ cells $\left./ \mathrm{mL}\right), 0.25 \% \mathrm{CNC} / \mathrm{PL}$, $0.25 \% \mathrm{CNC} / \mathrm{PL} \mathrm{w} / \mathrm{hDPCs}\left(4 \times 10^{6}\right.$ cells $\left./ \mathrm{mL}\right)$, previously prepared in similar conditions as described in section 2.10, were implanted on the CAM. Positive control group for angiogenesis consisted on sterile filter paper discs (4 mm in diameter) (MN GF-3; Macherey-Nagel) which were also implanted on the CAM at day 10 of embryonic development. Following implantation, shell opening windows were protected again with transparent tape and the eggs returned to the incubator at $37^{\circ} \mathrm{C}$ until day 14 of embryonic development. All these procedures were performed in a laminar flow hood to minimize contamination. CAM and underlying embryos were fixed in ovo with freshly prepared $4 \%(\mathrm{v} / \mathrm{v})$ formalin (Thermo Scientific) and subsequently incubated at $-80^{\circ} \mathrm{C}$ for 10 $\mathrm{min}$ in an ultra-low freezer. The implanted materials and underlying CAM portions were harvested and transferred to 6 -well plates containing the same fixative solution. Ex ovo images were acquired at $6.5 \times$ magnification using the AxioVision imaging software (release 4.8; Zeiss) connected to an AxioCAM ICc1 digital camera (Zeiss) attached to a stereomicroscope (Stemi 2000C; Zeiss). The excised membranes were transferred to histological cassettes, embedded in paraffin and serially sectioned in $4 \mu \mathrm{m}$-thick sections using a microtome (Rotary Microtome HM355S, MICROM International $\mathrm{GmbH}$, Walldorf, Germany). Three independent CAM assays were performed.

\subsubsection{Blood vessels convergence analysis}

The macroscopic evaluation of the angiogenic response was based on a semiquantitative method described by Silva-Correia et al. [31] consisting on the ex ovo analysis of blood vessels convergence toward the implanted discs. The ex ovo images obtained at day 14 of embryonic development were processed using the WCIF ImageJ software program (US National Institutes of Health) and the total number of convergent macroscopic blood vessels was blindly counted by three independent observers using a minimum number of 8 samples per tested condition. For quantification purposes, the stereomicroscope images were acquired at a constant magnification $(6.5 \times)$, and the image-processed area was maintained $(800 \times 800$ pixels $)$.

\subsubsection{Immunohistochemistry}

The CAM histological sagittal $4 \mu \mathrm{m}$-thick sections were stained with hematoxylin solution (Modified Mayer's Hematoxylin; Richard-Allan Scientific) and images were acquired under transmitted microscopy using a Microscope Leica DM750 (Leica; Germany) attached to a digital camera DMC5400 (Leica, Germany) connected to Leica Application Suite imaging software (release 4.6; Leica).

Representative paraffin embedded CAM $4 \mu \mathrm{m}$-thick sections were also submitted to immunohistochemical assay with SNA-lectin using the streptavidin-biotin peroxidase complex kit (UltraVision Large Volume Detection System Anti-Polyvalent, HRP; Lab Vision, Thermo Scientific, CA, USA) to detect chick origin endothelial cells. Briefly, CAM sections were deparaffinized and rehydrated in a decreasing series of ethanol (Fisher Chemical, USA). Then, the samples were exposed to heat-induced antigen retrieval with $10 \mathrm{mM}$ citrate buffer ( $\mathrm{pH} \mathrm{6}$; Merck) for 20 min at $98^{\circ} \mathrm{C}$. Endogenous peroxidases were blocked by incubation on a $3 \%(\mathrm{v} / \mathrm{v})$ hydrogen peroxide solution $\left(\mathrm{H}_{2} \mathrm{O}_{2}\right.$; Panreac Química SLU) for $10 \mathrm{~min}$ and washed with PBS (Sigma-Aldrich, USA). CAM sections were incubated in protein blocking solution (Ultra $V$ block; Lab Vision, Thermo Scientific, CA, USA) for 10 min followed by incubation with the primary antibody raised against lectin (SNA-lectin); Vector Laboratories, Burlingame, CA, USA) for $1 \mathrm{~h}$ at RT. CAM sections were sequentially washed with PBS and incubated with the streptavidin-peroxidase complex for $10 \mathrm{~min}$. The immune reaction was noted using 3,3'-diaminobenzidine solution (DAB; Vector Laboratories, Burlingame, USA) as chromogen. The counterstained was performed with Modified Mayer's Hematoxylin. The histological sections were observed, and images were acquired at different magnifications using Microscope Leica DM750 attached to a digital camera DMC5400.

The distinction of the different cells present in the hydrogel/ CAM sections was assessed and studied by staining the hDPCs and vascular cells with specific cells markers. To this end, representative CAM histological sagittal sections ( $4 \mu \mathrm{m}$-thick) were firstly deparaffinized and rehydrated in a decreasing series of ethanol (Fisher Chemical, USA). Then, the samples were submitted to a heat-induced antigen retrieval with pre-heated $10 \mathrm{mM}$ citrate buffer ( $\mathrm{pH} \mathrm{6}$; Merck, Germany) for $2 \mathrm{~min}$, and allowed to cool for $20 \mathrm{~min}$ at room temperature. CAM sections were washed twice for $5 \mathrm{~min}$ in $1 \times$ tris-buffered saline (TBS) buffer (100 mM tris base (Nzytech, Portugal), $150 \mathrm{mM} \mathrm{NaCl,} \mathrm{pH} \mathrm{7.5)}$ with $0.025 \%$ Triton X-100 (Sigma-Aldrich, Germany) and blocked in $10 \%$ normal donkey serum (Sigma-Aldrich, USA) with $1 \%$ BSA in TBS for $1 \mathrm{~h}$ at room temperature. Then the slides were incubated in the primary antibody overnight at $4{ }^{\circ} \mathrm{C}$, to immunostained the vessels of the CAM against mouse $\alpha$-smooth muscle actin ( $\alpha$-SMA, ab18147, Abcam, UK; 1:100) diluted in TBS with $1 \%$ BSA. Then, all samples were washed twice with TBS/0.025\% Triton X-100 for $5 \mathrm{~min}$, and incubated with the fluorescentlabelled secondary antibody alexafluor 488 donkey anti-mouse (A21202, Invitrogen, USA; 1:1000) diluted in TBS with 1\% BSA, at room temperature for $1 \mathrm{~h}$ and prevented from light exposure. After rinsing twice with TBS/0.025\% Triton X-100 for $5 \mathrm{~min}$, the human mesenchymal cells encapsulated into the hydrogels were immuno-stained with CD90 (mouse anti-human CD90 APC, \#559869, Pharmingen, USA; 1:100) at room temperature for $1 \mathrm{~h}$. Then, samples were washed 3 times with TBS for $5 \mathrm{~min}$ to remove remaining residues and the cells nuclei were counterstained with DAPI $1: 1500 \mathrm{v} / \mathrm{v}$ for $1 \mathrm{~h}$ at room temperature. After rinsing in TBS to reduce background fluorescence, immunolabeled samples were mounted and analysed by transmitted and reflected light microscope (Axio Imager Z1m, Zeiss, Germany). Each experiment was performed in duplicate. 


\section{Statistical analysis}

The statistical analysis of data was performed using GraphPad PRISM version 6.0 (GraphPad Software Inc., CA, USA). One- and two-way analysis of variance (ANOVA) was performed, followed by the Tukey posthoc test or the Bonferroni post-test to assess significant differences, respectively. Statistical significance and associated degree of confidence $(\mathrm{p}<0.05)$ are represented by symbols stated in the graphs. Results are presented as mean \pm standard error of mean.

\section{Results}

\subsection{In situ gelation time}

Stable hydrogels were quickly formed upon cross-link reaction of the precursor components, regardless the formulation (Fig. 2A). The measurements of the apparent in situ gelation time of the hydrogels are depicted in the hydrogels with and without PL. HA/CNCs hydrogels presented gelation time values in the range of $2.7 \pm 0.6$ to $19.3 \pm 2.1 \mathrm{~s}(0.5 \% \mathrm{CNC}-0 \% \mathrm{CNC})$, while the values obtained for PL-based hydrogels were between $3.3 \pm 0.6$ and 127 $\pm 31.3 \mathrm{~s}$ for $0.5 \% \mathrm{CNC} / \mathrm{PL}$ and $0 \% \mathrm{CNC} / \mathrm{PL}$, respectively. In this sense, the increase of a-CNCs content resulted in the decrease of the working gelation time $(p<0.05)$. On the other hand, the incorporation of PL did not have a significant effect, with the exception of the formulation $0 \% \mathrm{CNC} / \mathrm{PL}$ which presented the higher gelation time $(127 \pm 31.3 \mathrm{~s} ; \mathrm{p}<0.05)$.

\subsection{Hydrogel microstructure}

The influence of a-CNCs and PL incorporation on hydrogels' microstructure were investigated by cryo-SEM. Representative images of the analyzed cross sections are depicted in Fig. 2C. The pore sizes tend to increase with the incorporation of increasing ratios of a-CNCs, peaking for the formulation incorporating $0.25 \%$ a-CNCs (Fig. 2D). Therefore, in the formulations without PL, the mean pore size ranged from 1.9 to $9.4 \mu \mathrm{m}$ for $0 \% \mathrm{CNC}$ and $0.25 \%$ CNC, respectively.

The incorporation of PL resulted in a relatively more organized and compact structure, presenting a more homogeneous porous microstructure with significantly smaller pores, ranging from 0.9 to $3.3 \mu \mathrm{m}$ for $0 \% \mathrm{CNC} / \mathrm{PL}$ and $0.25 \% \mathrm{CNC} / \mathrm{PL}$, respectively.

\subsection{Assessment of the mechanical/viscoelastic properties of hydrogels}

The mechanical/viscoelastic properties of the hydrogels were tested by rheology analysis in a physiological-like environment. The results are presented in Fig. 3 where the elastic modulus $\left(\mathrm{G}^{\prime}\right)$-time and the loss factor $(\tan \delta$ )-time of all the groups, at the frequency of $1 \mathrm{~Hz}$, are shown. Hydrogels without PL, exhibited maximum $\mathrm{G}^{\prime}$ values that ranged between 410 and $965 \mathrm{~Pa}$, for $0 \%$
A)

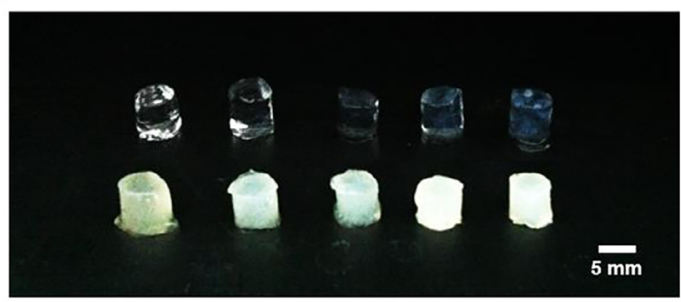

B)

\section{$0 \% \mathrm{CNC} \quad 0.25 \% \mathrm{CNC} \quad 0.5 \% \mathrm{CNC}$}

\begin{tabular}{cccc}
\hline No PL & $19.3 \pm 2.1 \mathrm{~s}$ & $9.7 \pm 1.5 \mathrm{~s}$ & $2.7 \pm 0.6 \mathrm{~s}$ \\
\hline PL & $127 \pm 31.3 \mathrm{~s}$ & $4.7 \pm 0.6 \mathrm{~s}$ & $3.3 \pm 0.6 \mathrm{~s}$ \\
\hline
\end{tabular}

C)

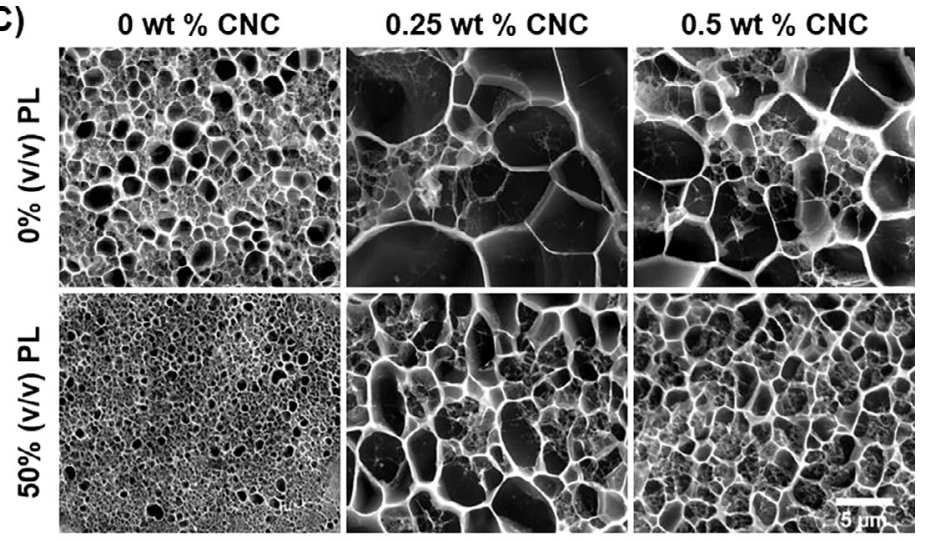

D)

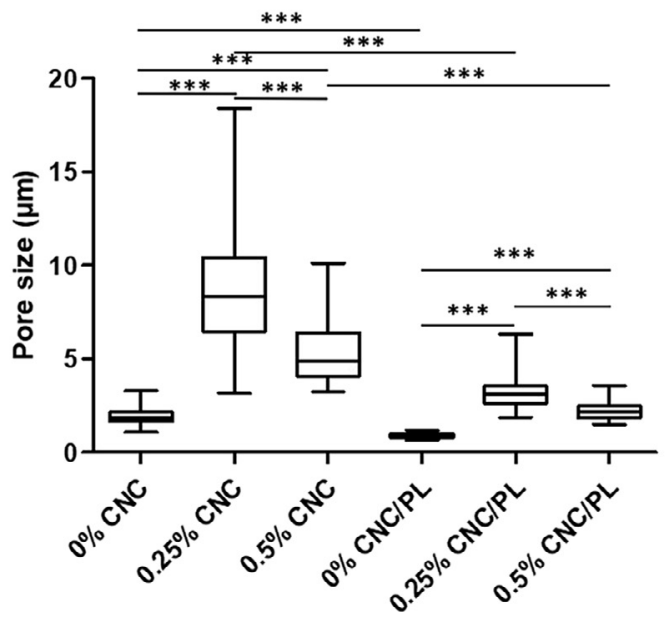

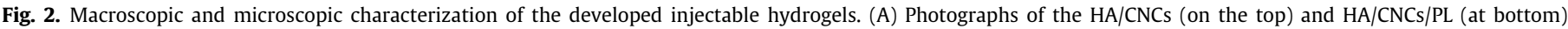

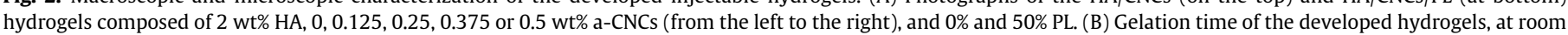

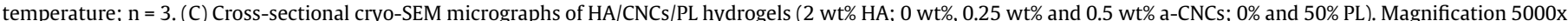

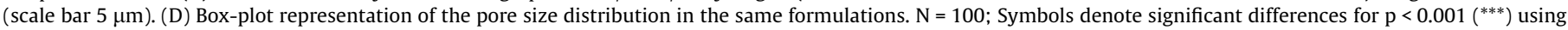
One-Way ANOVA followed by the Tukey posthoc test for multiple comparisons. 

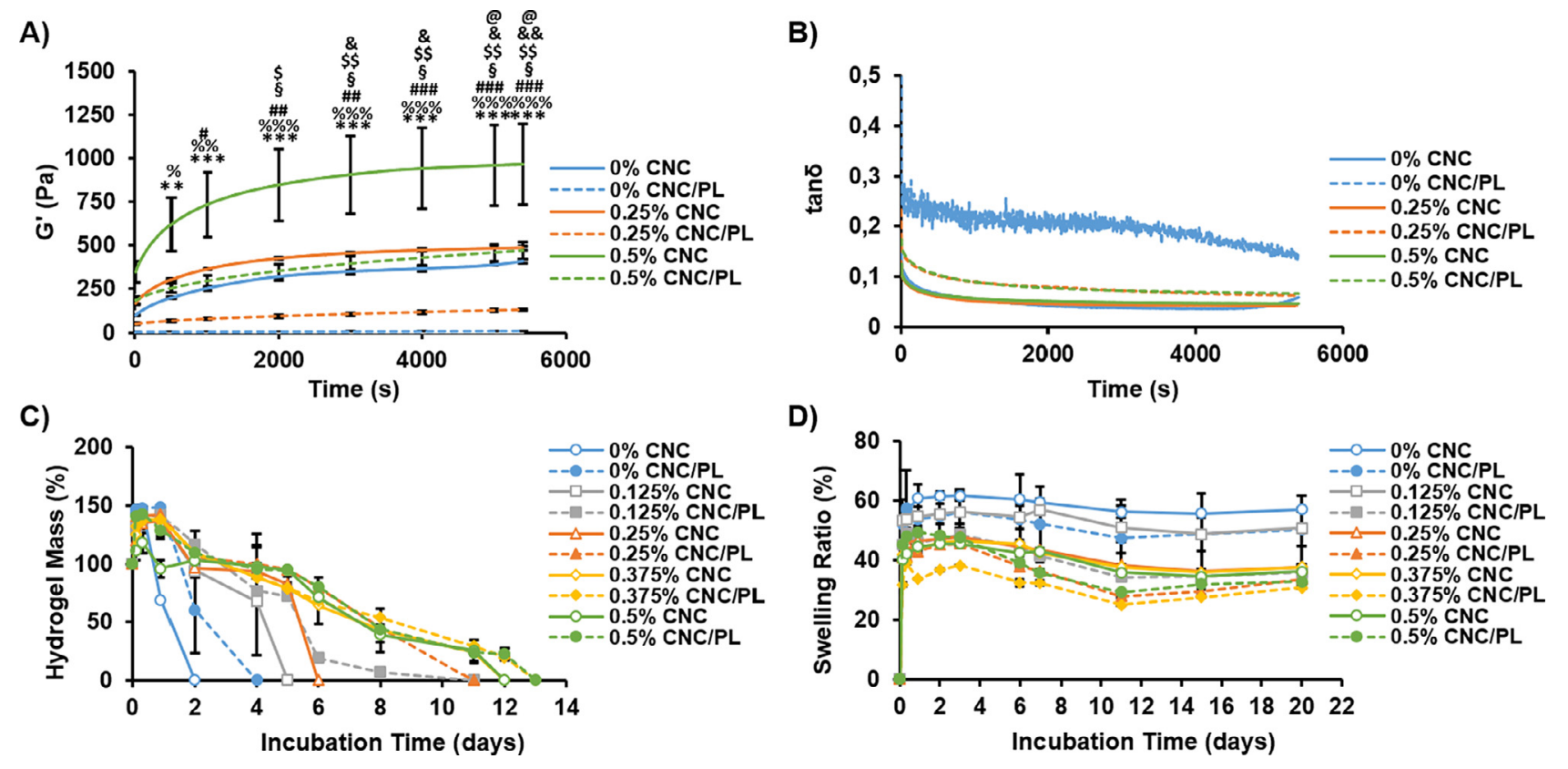

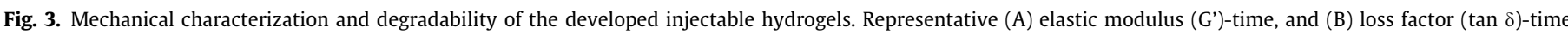

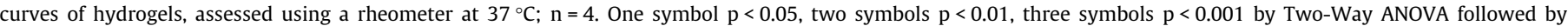

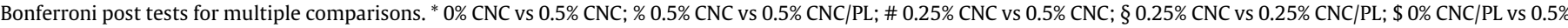

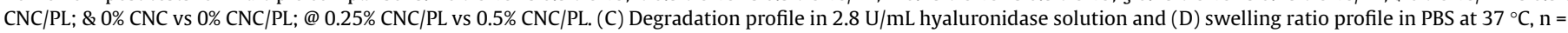
5. Statistical analysis described in the supporting information.

CNC and $0.5 \%$ CNC formulations, respectively (Fig. 3A). Hydrogels enriched with PL, exhibited $\mathrm{G}^{\prime}$ values of 9 and $471 \mathrm{~Pa}$ for a-CNCs content 0 and $0.5 \mathrm{wt} \%$, respectively. These results show that the incorporation of a-CNCs in the HA matrix lead to stiffer hydrogels (higher $\mathrm{G}^{\prime}$ ) indicating that they induce a reinforcement effect by acting as effective junction elements. Moreover, PL-laden hydrogels presented lower $G^{\prime}$ values in comparison with formulations without PL, thus, presenting lower stiffness and higher elasticity.

The $\tan \delta$ represents the ratio between the amount of energy dissipated by viscous mechanisms $\left(\mathrm{G}^{\prime \prime}\right)$ and the energy stored in the elastic component $\left(\mathrm{G}^{\prime}\right)$, giving information about the damping properties of the material. In the last data point, hydrogels without PL, exhibited $\tan \delta$ values that ranged between 0.04 and 0.06 , for $0.25 \% \mathrm{CNC}$ and $0 \% \mathrm{CNC}$ formulations, respectively (Fig. 3B). Hydrogels enriched with PL, exhibited $\tan \delta$ values of 0.06 and 0.1 for aCNCs content 0.25 and 0.5 wt\%, respectively. Despite of PL-laden hydrogels present slightly higher values, the overall low values (near zero) are indicative of the highly elastic properties of the hydrogels and low energy dissipating potential.

\subsection{Enzymatic degradation and swelling assays}

The degradation profile of the hydrogels was obtained as a function of incubation time in HAase solution, as shown in Fig. 3C. The statistical analysis is depicted in the supporting information, Table S1. Overall, the obtained results showed that the incorporation of both a-CNCs and PL in HA hydrogels influences their stability. All hydrogel formulations swelled during the first $8 \mathrm{~h}$ of incubation. After this point in time, some samples ( $0 \% \mathrm{CNC}, 0.5 \%$ $\mathrm{CNC}, 0.5 \% \mathrm{CNC} / \mathrm{PL}$ ) started undergoing enzymatic degradation, while the remaining samples swelled up to $22 \mathrm{~h}$ of incubation. The incorporation of CNCs in the hydrogels matrix increased their resilience to enzymatic degradation; the hydrogels without CNCs totally degraded after 2 days of incubation, while the formulations with increasing concentrations of nanoparticles degraded only after 5, 6 and 12 days, respectively. Regarding the PL-loaded hydro- gels, samples without CNCs completely dissolved after 4 days, while formulations with increasing CNCs content degraded only after 11 and 13 days of incubation.

The swelling profile over time was followed up to 21 days of incubation in PBS at $37{ }^{\circ} \mathrm{C}$, as depicted in Fig. 3D, and the statistical analysis is presented in the supporting information (Table S2). Hydrogels with higher content of a-CNCs and with/without PL ( $0.5 \mathrm{wt} \% \mathrm{CNC} / \mathrm{PL}$ and $0.5 \mathrm{wt} \% \mathrm{CNC}$ ) reached equilibrium within $22 \mathrm{~h}$ and 2 days of incubation period and presented a mass increase of $49.5 \pm 0.99 \%$ and $45.52 \pm 0.33 \%$, respectively. After 2-3 days of incubation, all the hydrogels had reached the swelling equilibrium. The incorporation of both CNCs and PL decreased the maximum swelling, with the formulations $0 \% \mathrm{CNC}, 0 \% \mathrm{CNC} / \mathrm{PL}$ and $0.125 \%$ CNC presenting a maximum mass increase between 56.30 $\pm 7.40 \%$ and $61.68 \pm 1.00 \%$, while the remaining formulations presented a maximum swelling between $38.24 \pm 0.18 \%$ and $47.98 \pm$ $0.98 \%$. After this point in time, hydrogels' weight remained approximately unaltered during the 21 days follow up.

Overall, the results from enzymatic degradation and swelling assays showed that incorporation of both a-CNCs and PL into the HA hydrogels reduced their degradation behavior and swelling ability.

\subsection{Release of pro-angiogenic platelet lysate-origin growth factors}

The release of pro-angiogenic GFs from the developed PL-laden hydrogels incubated in PBS or $2.8 \mathrm{U} / \mathrm{mL}$ HAase was quantified by ELISA. The amount of GFs released from the hydrogels incubated in PBS was not detectable by ELISA. Nevertheless, the enzymatic degradation of the hydrogels allowed the release of PL-origin GFs. The results for the release of VEGF, depicted in Fig. 4A, showed that the different hydrogels displayed a similar release profile characterized by an initial "burst" of protein released up to day 2. After 2 days, the release kinetics reached an apparent plateau, and a slow and sustained delivery remained up to the maximum release time of each formulation, with exception of the $0 \%$ 
A)

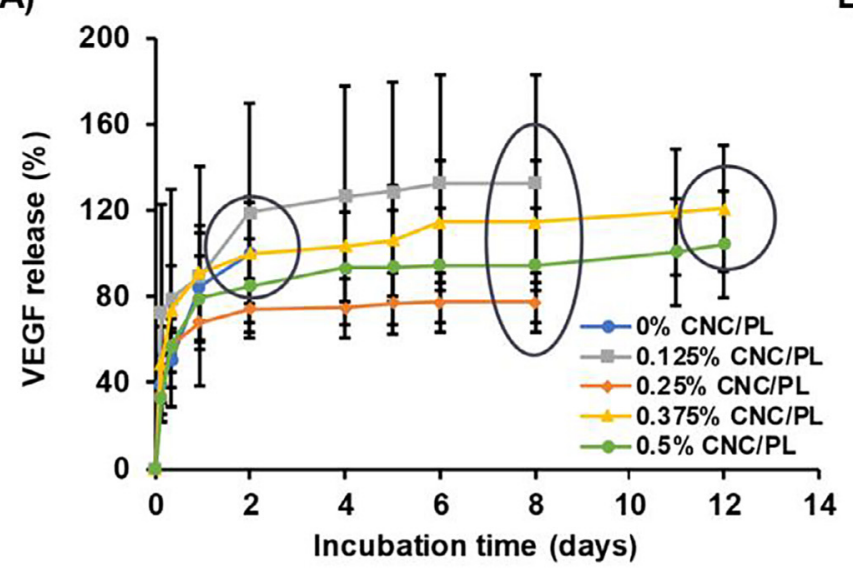

B)

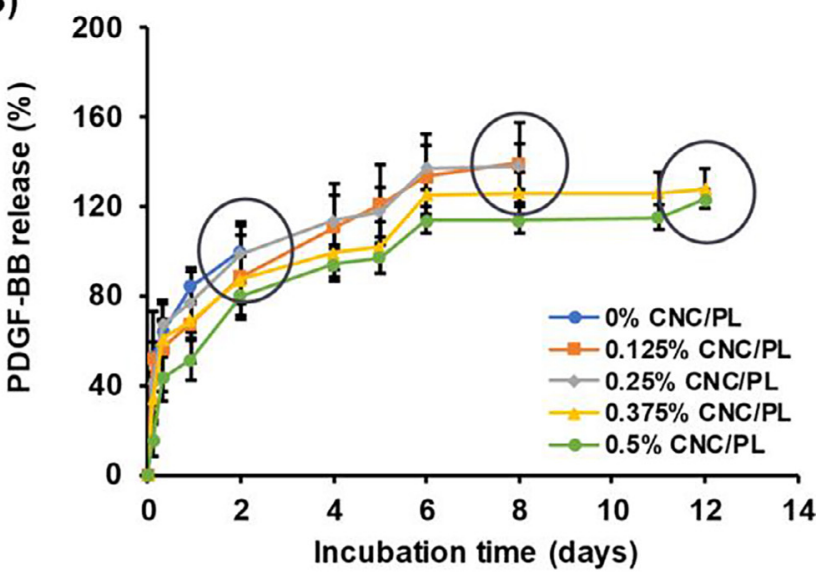

C)

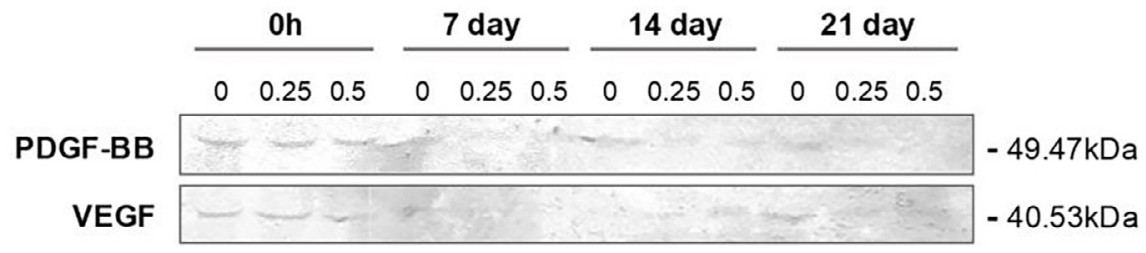

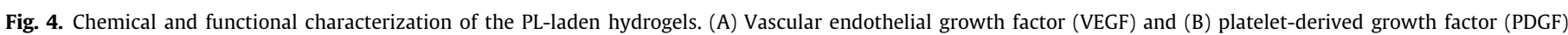

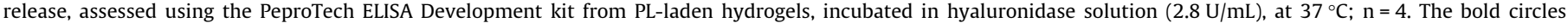

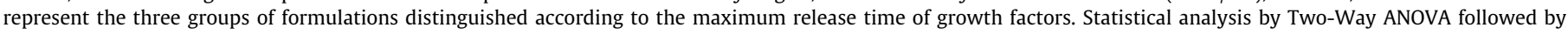

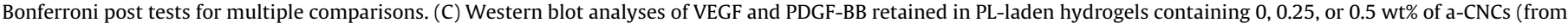
the left to the right), incubated in basal medium for 21 days.

$\mathrm{CNCs} / \mathrm{PL}$ samples that ceased the release at day 2 . Regarding the release of PDGF-BB, depicted in Fig. 4B, the profiles showed a sustained release, progressing in a linear way up to day 2 for $0 \% \mathrm{CNC} /$ PL hydrogels and day 6 for the remaining formulations. After day 6 , the release profiles reached an apparent plateau.

Overall, the profiles demonstrated a slower release of both GFs. Although no statistically significant differences were observed between the formulations, there was a significant difference in the maximum release time, which is proportional with the amount of CNCs incorporated in the formulations. In this sense, three groups of formulations were distinguished according to the maximum release time of GFs: $0 \% \mathrm{CNC} / \mathrm{PL}$ hydrogels released up to day 2 ; $0.125 \%-0.25 \%$ CNC/PL samples released for 8 days; and $0.375 \%-$ $0.5 \% \mathrm{CNC} / \mathrm{PL}$ hydrogels released up to day 12 .

The GFs stability within the hydrogels was also studied over the incubation time in basal medium by Western Blotting. The results demonstrated the presence of the specific bands for each GF in study (Fig. 4C); the dimer of PDGF-BB (49.47 kDa) and the Isoform L-VEGF165 (40.53 kDa) were present in the majority of the formulations and time points, with a similar intensity of the bands which slightly decreased with the incorporation of a-CNCs.

\subsection{Encapsulation of human dental pulp cells}

The ability of hydrogels to support cell adhesion and proliferation and control biochemical and cellular mechanisms is one of the requirements for TE applications. To examine the capability of the developed hydrogels enriched with PL to entrap cells and support their viability, the quantity of DNA and the metabolic activity were evaluated after $1,3,7$, and 14 days of culturing. In both the cases, the incorporation of PL in the hydrogels had a positive effect in the cells proliferation rate and metabolic activity as shown in Fig. 5.
Interestingly, the hDPCs encapsulated in hydrogels containing $0 \%$ of a-CNCs demonstrated higher cell content in almost all time points of culturing (Fig. 5A). However, the cytotoxic effect of CNCs over hDPCs seems to be overcome by the incorporation of PL. The PL-laden hydrogels presented much higher hDPCs content for all the time points. The positive effect of PL incorporation was also observed through the analysis of the metabolic activity per cell (Fig. 5B). Overall, the hDPCs remained viable, with constant metabolic levels per cell, throughout the experiment for samples containing PL. In contrast, the formulations without PL presented a considerable reduction on cell viability, with peaks of metabolic activity per cell after 7 and 14 days of incubation, for the $0 \% \mathrm{CNC}$ and $0.25 \% \mathrm{CNC}$ formulations.

Regarding the cells morphology and cytoskeleton organization, the results shown that hDPCs encapsulated into the hydrogels with incorporated PL were spread within the hydrogel and exhibited elongated shapes, with pronounced and extended actin filaments after 7 days of culturing, while only disparate round-shaped and dispersed cells were found on samples produced without PL (Fig. 5C). Moreover, 3D reconstruction based on confocal imaging showed not only the presence of a homogeneous distribution of the cells in both hydrogels with and without PL but also welldeveloped multidimensional networks of cell-to-cell and cellmatrix contacts throughout the PL-laden hydrogels (Fig. 5D).

\subsection{In vitro evaluation of the chemotactic and proangiogenic properties of the HA/PL hydrogels}

To assess the effect of the PL-laden hydrogel on hDPCs invasion or sprouting in $3 \mathrm{D}$, cell pellets containing only hDPCs or a $1: 1$ ratio of CM-Dil-labeled hDPCs and CFSE-stained HUVECs were stained and embedded into the hydrogels, and their respective invasion/ 
A)

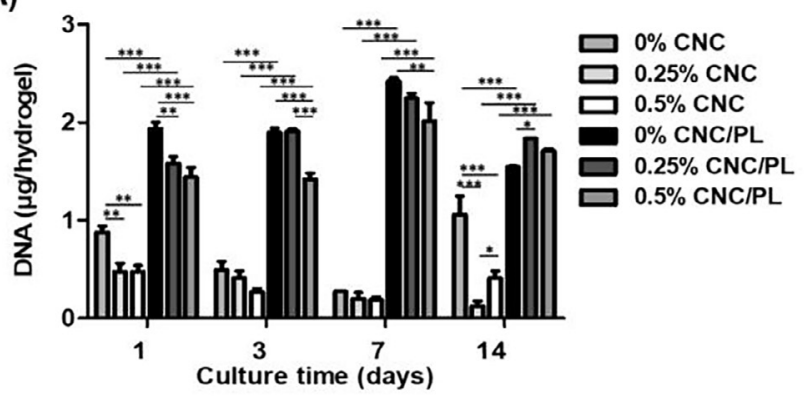

C)

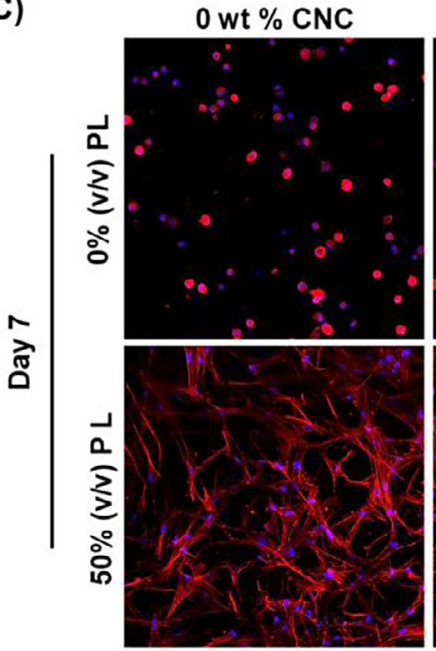

B)

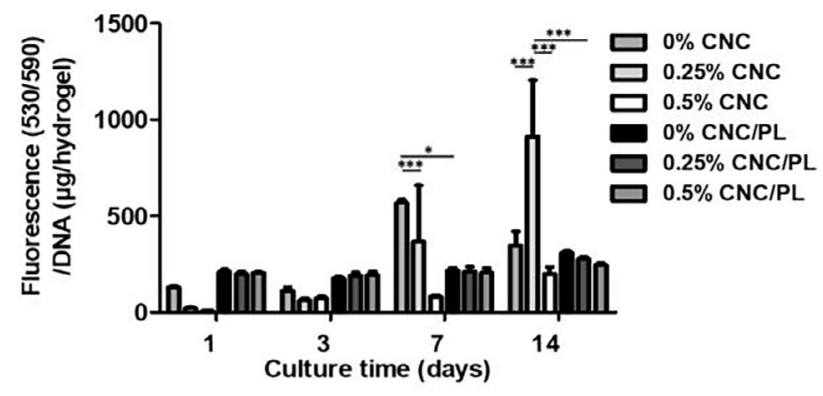

0.25 wt $\%$ CNC

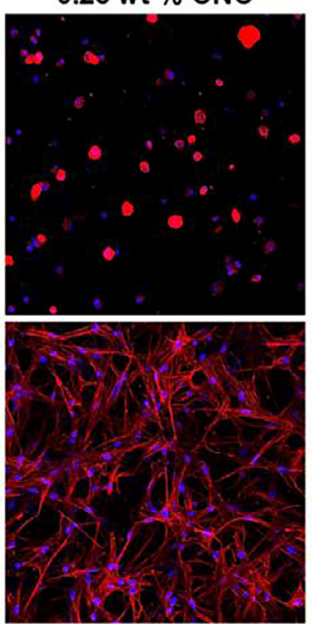

0.5 wt $\%$ CNC
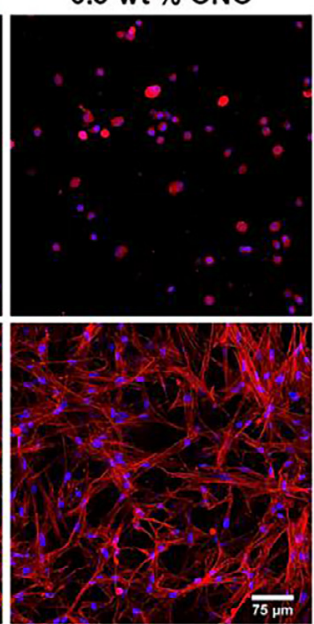

0.25 wt $\%$ CNC

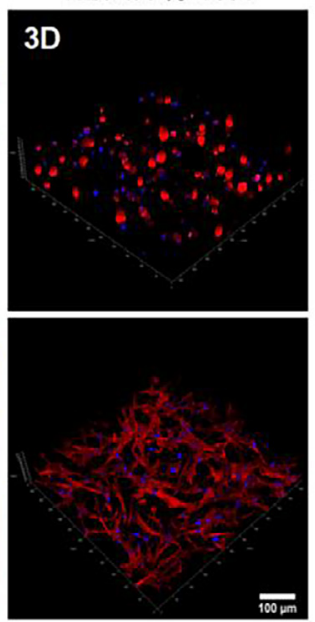

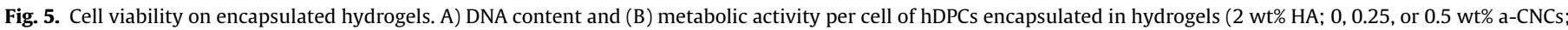

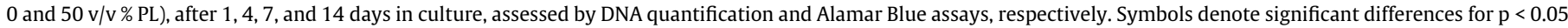

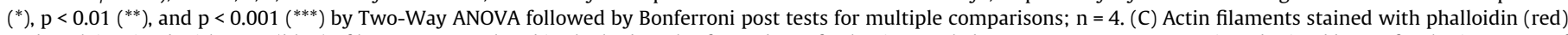

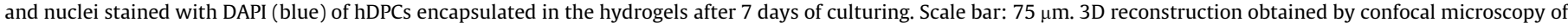

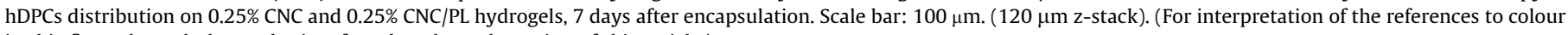
in this figure legend, the reader is referred to the web version of this article.)

sprout length was quantified over 3 days (Fig. 6A). The sprout length from hDPCs pellets invading the PL hydrogel increased throughout the course of the experiment, conversely to pellets in HA/CNCs hydrogels (Fig. 6B and C). Moreover, at each time point, the cells invasion was significantly greater in PL-laden hydrogels $(p<0.001)$. Likewise, there was also an increase in sprout length from co-culture pellets embedded in PL-laden hydrogels compared to HA/CNCs constructs at all 3 days ( $p<0.001)$ (Fig. 6D and E). Regarding PL-enriched hydrogels, cells invasion was increased in formulations containing $0.25 \%$ of a-CNCs over 3 days, both in monocultures of hDPCs and co-cultures. hDPCs demonstrated longer outward migration from co-cultures (173.52 $\pm 5.49 \mu \mathrm{m}$ for $0.25 \% \mathrm{CNC} / \mathrm{PL}$ formulation) when compared to pellets only containing hDPCs ( $156.38 \pm 10.84 \mu \mathrm{m}$ for $0.25 \% \mathrm{CNC} / \mathrm{PL}$ formulation).

The cells morphology and arrangement in the pellet, was also analysed after the 3 days of culturing, where pellets were stained with specific antibodies against mesenchymal and endothelial cells (CD90 and CD106, respectively). Since a more pronounced cell sprouting was evidenced in all PL-laden hydrogels samples, the morphology and distribution of the encapsulated cells was only assessed for these hydrogels.

The confocal images showed that the sprouts were mainly composed of hDPCs, exhibiting spindle-like morphology and extensive spreading (Fig. 6F, H). This type of cell behaviour was even more pronounced in co-cultures, especially in hydrogels formed with $0.25 \%$ of a-CNCs (Fig. $6 \mathrm{G}$ ). Moreover, the monocultures of hDPCs presented centrifugal individual migration of cells while in cocultures the sprouts were composed of side by side cells forming tubular-like structures. Regarding the HUVECs contribution for the sprouting, confocal micrographs showed that $\mathrm{CD} 106^{+}$cells were confined to the centre of the pellet and rarely participating in the formation of the sprouts (Fig. 6F, H). These results were also confirmed with the analysis of sprouts morphology obtained by $3 \mathrm{D}$ reconstruction based on confocal imaging, available in: https:// youtube/M2b1iMOklJQ.

\subsection{Ex vivo evaluation of the HA/PL hydrogels ability to promote neo-} vascularization

To confirm the ability of the injectable system to promote angiogenesis, an ex vivo CAM assay was performed. The quantification of convergent macroscopic blood vessels (Fig. 7A) demonstrated that there were no significant differences among the different HA-based formulations tested. However, it was possible to notice that the filter paper (positive control) exhibited a significant higher number of convergent macroscopic blood vessels comparing to the different HA formulations, being this difference less pronounced for the formulations 0.25\% CNC/PL and $0.25 \%$ $\mathrm{CNC} / \mathrm{PL} \mathrm{w} / \mathrm{hDPCs}$.

In Fig. $7 \mathrm{~B}$ it is depicted the light microscopy images of the excised CAM sagittal sections with the implants, after 4 days of implantation and stained with hematoxylin and SNA-lectin. 
A)

1

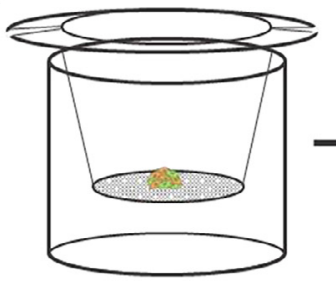

2

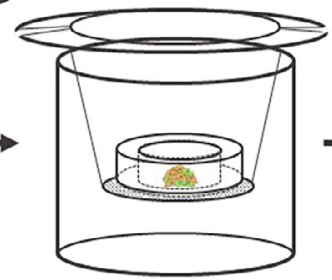

3

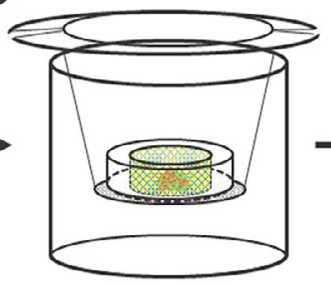

4

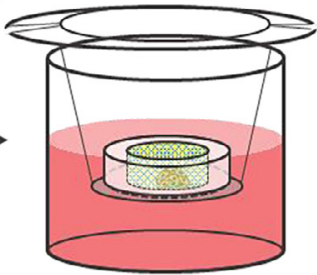

B)

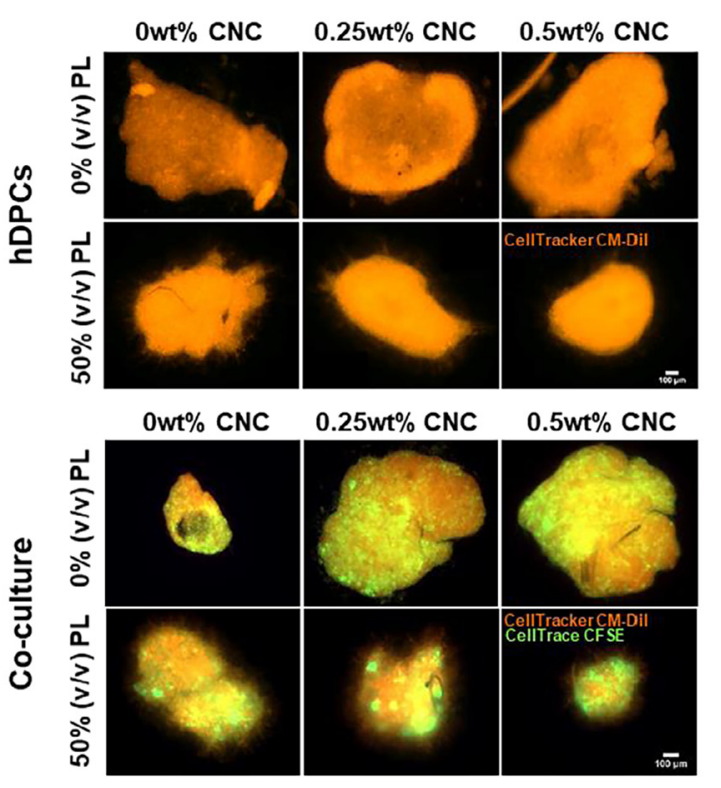

F)

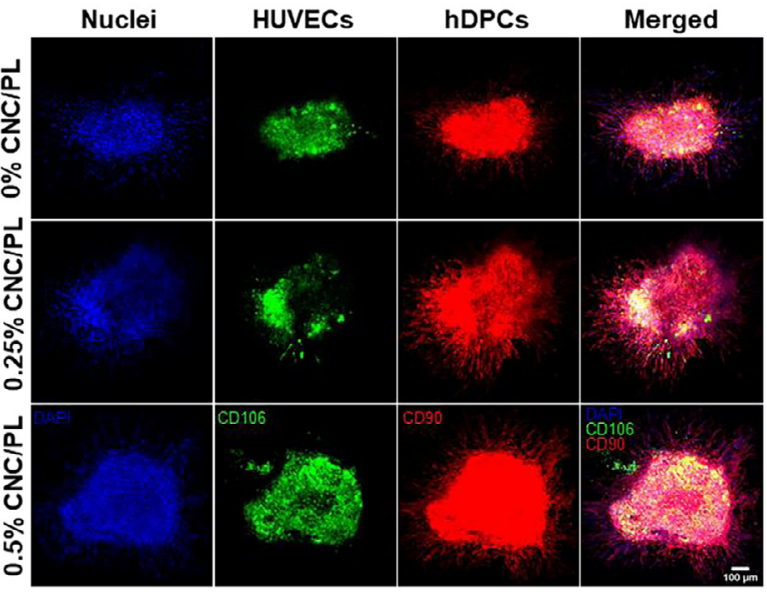

C)

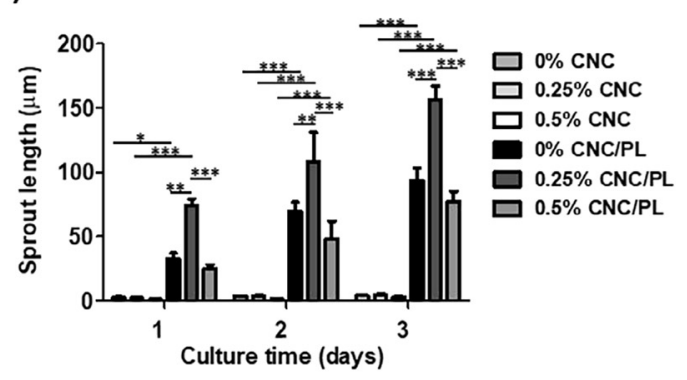

E)

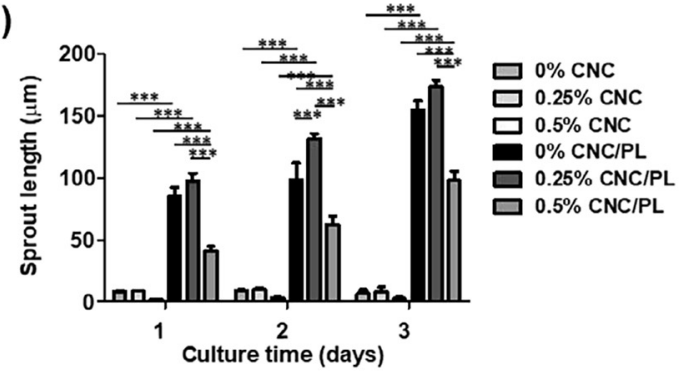

G)

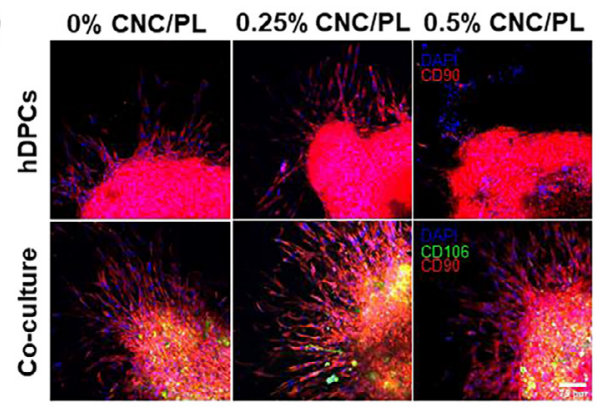

H)

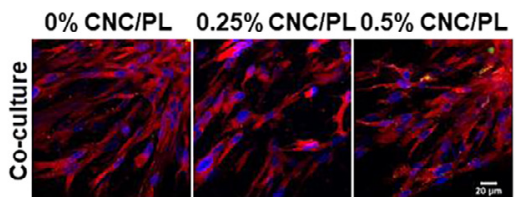

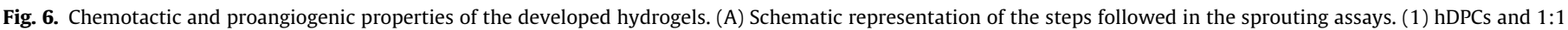

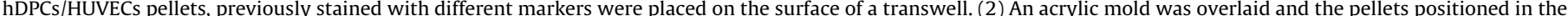

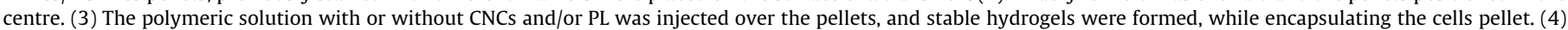

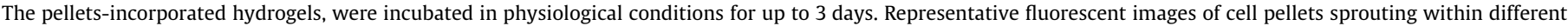

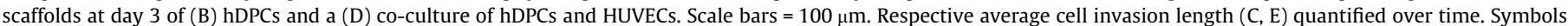

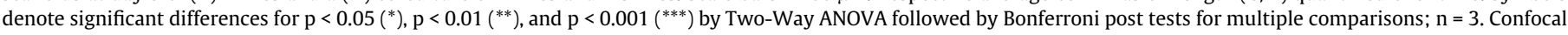

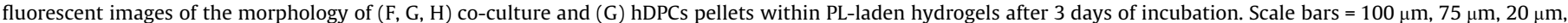
respectively.

Histological characterization showed no evidence of absorption of any of the tested materials. Also, it was observed that the materials did not elicit an acute inflammatory response, since there was no formation of granulation tissue. Moreover, it was observed that in formulations $0 \% \mathrm{CNC}, 0 \% \mathrm{CNC} \mathrm{w} / \mathrm{hDPCs}$, and $0.25 \% \mathrm{CNC} / \mathrm{PL}$ there is an interface between the hydrogels and the CAM, but no chick endothelial cells infiltration occurred. Contrarily, in the $0.25 \%$ $\mathrm{CNC} / \mathrm{PL} \mathrm{w} / \mathrm{hDPCs}$ hydrogels it was possible to notice a massive 
A)

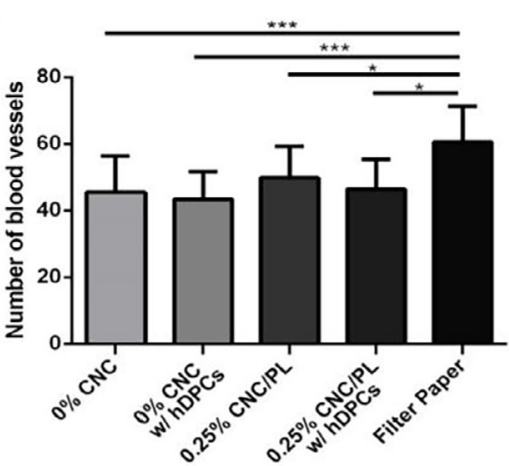

B)
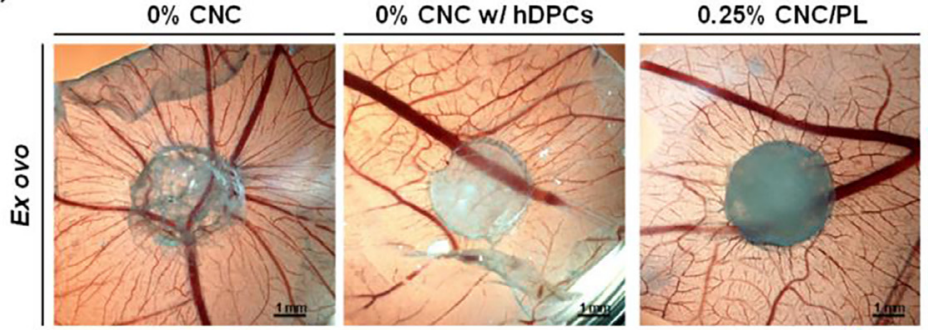

$0.25 \%$ CNCIPL w/ hDPCs

Filter paper
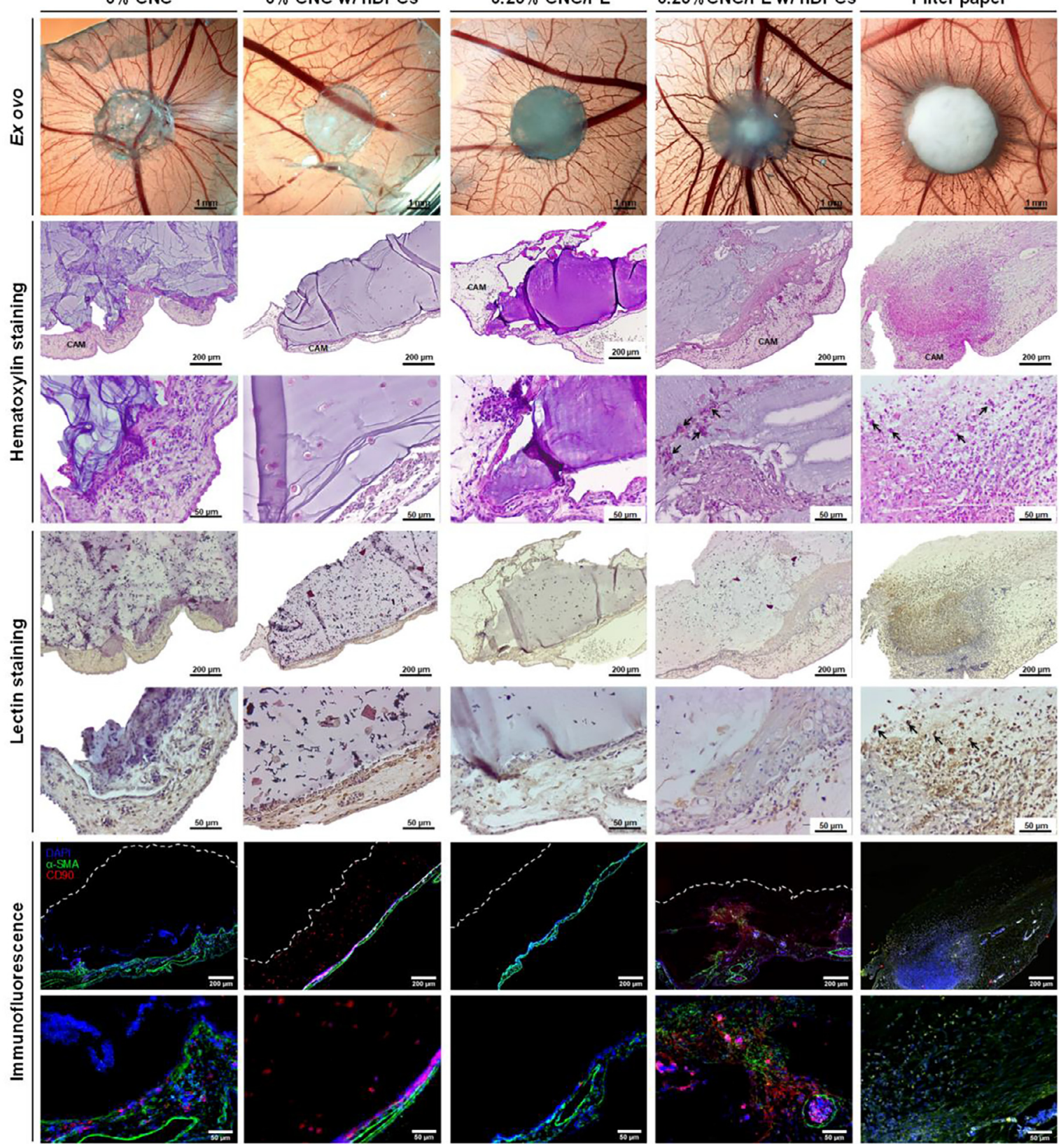

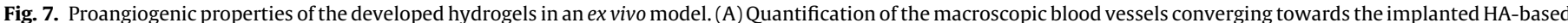

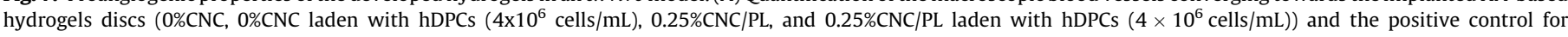

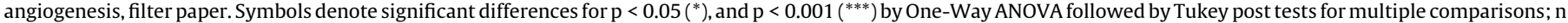

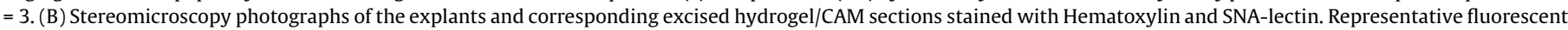

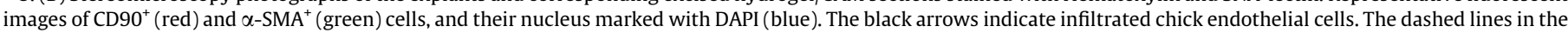
fluorescent images delimit the hydrogels. (For interpretation of the references to colour in this figure legend, the reader is referred to the web version of this article.) 
infiltration of surrounding tissue and chick erythrocytes and a reorganization of hDPCs, which apparently seems to converge to the formation of microscopic blood vessels.

Regarding the immunofluorescent images it is visible the expression of $\alpha$-SMA by the cells in the CAM blood vessels walls and in the cell mass infiltrating the $0.25 \% \mathrm{CNC} / \mathrm{PL}$ w/hDPCs hydrogels or the filter paper. In the $0 \% \mathrm{CNC} \mathrm{w} / \mathrm{hDPCs}$ is visible the homogeneous distribution of scattered human $\mathrm{CD}^{+} 0^{+}$cells. Remarkably, the cells infiltrating the $0.25 \% \mathrm{CNC} / \mathrm{PL}$, arranging in the vicinity of new primordial microscopic vessels, represented by the circulararranged red blood cells within the hydrogel, and expressing $\alpha$ SMA were human $\mathrm{CD}^{+} 0^{+}$. Together, the results suggest that the addition of PL can have a chemotactic effect, thus potentiating the migration of cells and formation of new convergent blood vessels.

\section{Discussion}

The present work describes the amelioration of a class of injectable HA-based hydrogels, reinforced with CNCs by incorporation of PL, aiming the augmentation of chemotactic and pro-angiogenic activity for the regeneration of vascularized injured tissues. The combination of $\mathrm{HA}, \mathrm{CNCs}$ and $\mathrm{PL}$, as herein proposed, produced an in situ cross-linkable system with several advantages for TE applications. Being injectable and cross-linked in situ, the system can easily fit to irregular-shaped defects, while deeply interact with the preserved tissues. Moreover, it can be administrated using minimally invasive techniques without requiring surgical interventions. HA-based biomaterials have demonstrated positive results for several potential applications in the regeneration of soft tissues [32], due to its natural similarity to ECM, and important role in several cellular processes including, cell proliferation, morphogenesis, inflammation, and wound repair [33]. CNCs have been employed as nanofillers or crosslinkers in biobased strategies, resulting in composite materials with improved mechanical properties $[13,17]$. PL can be used in clinical applications as an autologous [34,35] or allogenic therapy [36-38]. Further than the extensive variability in the quality of platelet rich hemoderivative batches across donors reported by several authors [39-42], the autologous therapeutic administration of platelet rich hemoderivative is conditioned by the limitations in whole blood volume yield. The generation of PL through pooled platelet concentrates from ten donors, biological qualified for blood transfusion (Decreto-Lei No. $100 / 2011$ ), is expected to provide more consistent and reproducible clinical outcomes $[39,43]$.

With regard to the gelation time, the hydrogel formulations formed quickly upon mixing of the two HA derivatives and the aCNCs, a characteristic of several hydrazone cross-linked HA hydrogels which present typical gelation times within 3-30 s [17,44,45]. CNCs content showed a positive effect on the gelation time of the hydrogels; the decrease of gelation time was proportional to the amount of the available aldehyde moieties for cross-linking incorporated through a-CNCs. In live tissues, the excessive bleeding might promote polymeric solution dissolution instead of polymerization. Thus, the fast gelation time observed, while hindering the manipulation of the polymeric solution shortly after injection and the post-injection procedures, allows the formation of a stable matrix which can work as a hemostatic plug.

Regarding the viscoelastic properties, rheological analysis revealed that the incorporation of a-CNCs in the HA matrix lead to stiffer hydrogels, inducing a reinforcement effect by acting as effective junction elements. Previous studies on the reinforcement of gelatin hydrogels with a-CNCs [22] also reported a significant improvement in gelatin hydrogels crosslinked with a-CNCs compared to unfilled hydrogels. Hydrogel mechanical properties are important for the stability of the material in culture and may also influence cellular mechanotransduction, which in turn has consequences for cellular behaviors like spreading, migration, and stem cell differentiation [46]. With an increase in substrate stiffness, cells usually exhibit enhanced cell adhesion [47], spreading with defined actin organization [48], and proliferation [49]. Regarding stem cell differentiation, cells seem to be tuned mechanically (compliancematched) so that they preferentially differentiate on ECMs that have a mechanical stiffness similar to that of their natural tissues $[50,51]$. Thus, the incorporation of variable amounts of a-CNCs into the polymeric matrix may be a useful tool for tuning the hydrogel mechanical properties and cells behavior, according to the targeted tissue. Moreover, PL-laden hydrogels presented lower $\mathrm{G}^{\prime}$ values, thus, presenting higher elasticity. This may be explained by the higher degree of mobility provided by PL protein chemical integration within the polymeric matrix via amine-aldehyde cross-linking. The adipic acid hydrazide linker provides a shorter crosslinking distance and lower degree of mobility, therefore restricting the polymer chain mobility and increasing its stiffness.

The degradation of the developed hydrogels occurred in the presence of the HAase, the specific enzyme that naturally degrades HA in vivo [52,53]. It should be noted that in this study we used a supra-physiologic concentration of HAase, 100 -fold greater than in human plasma (approximately $2.8 \mathrm{U} / \mathrm{L}$ [54]), since the degradation rate of HA, mediated by HAase, can be increased in case of injury, and to ensure complete gel degradation before HAase inactivation (this enzyme remains active up to $5 \mathrm{~h}$ at $37^{\circ} \mathrm{C}[55]$ ). In this sense, these HA-based materials have the advantage to be degraded and release their content in a controlled manner, while providing time and space stability for new tissue ingrowth. As already shown in previous studies [13], the degradability of the hydrogels can be controlled through the incorporation of a-CNCs. The ratio of hydrazide/aldehyde in the $0 \%$ CNC formulation is noticeably $2: 1$, whereby the incorporation of a-CNCs will increase the degree of cross-linking of the hydrogel matrix, and consequently decrease the degradation rate. Remarkably, the PL-enriched hydrogels remained stable for longer periods than hydrogels without PL. Such reinforcement is attributed to the presence of fibrinogen in the PL $[30,56]$, as this protein is capable of cross-linking, forming a fibrin mesh which is not susceptible to degradation by the HAase. In addition, the amines of the PL proteins can by themselves react with the aldehyde groups by Schiff's base reaction, becoming an integral part of the matrix. These results are in line with previous studies reported by other authors for HA-based hydrogels $[25,45]$, demonstrating that the incorporation of CNCs and PL in the HA matrix could modulate their in vivo resorbable properties by native environment. Likewise, the swelling profiles showed a decreasing trend with increasing CNCs and PL content following the trend previously reported studies on polymeric hydrogels reinforced with CNCs $[17,22,57]$. In fact, the swelling profile of hydrogels largely depends on the hydrophilicity of their functional groups and the cross-linking density of the polymeric matrix [57], which, as previously discussed, increase with a-CNCs and PL incorporation. Soft tissue defects must be reliably restored to their original shape and dimensions in addition to having physiological function. Swelling property of the hydrogels is crucial for substance exchange; however, an exceeding swelling behaviour can result in postoperative volume augmentation and displacement from the implantation site $[58,59]$. In endodontic regenerative therapies, this feature can increase the hydrostatic pressure inside the root canal system, thus breaking the tooth from contact areas between cement/resin and dentin/enamel or from existing clefts in the tooth. Compared to high swelling HA-based hydrogels $[60,61]$, the lower swelling of the developed bionanocomposite hydrogels makes them suitable to accurately retain the shape and form of the final tissue structure. 
The release of PL-origin chemotactic and proangiogenic factors PDGF-BB and VEGF from the hydrogels, was detected only after degradation of the hydrogels in HAase, whereas no detectable traces of GFs were detected after incubation of the hydrogels in PBS. The desirable sustained release of GFs identified here may be due to their sequestration by the microstructural networks as well as the result of resistance of PL to autolysis. Moreover, the release of both GFs was proportional with the amount of incorporated CNCs. As mentioned before, the incorporation of CNCs lead to a higher crosslinking density, and consequently to a lower degradation rate, which resulted in a slower and longer release of the GFs by prolonging the entrapment time of the GFs. On the other hand, the GFs, usually positively charged at physiologic $\mathrm{pH}$, bind electrostatically to negatively charged polymers. It is known that sulfated glycosaminoglycans have the ability to noncovalently bind and modulate cytokines, GFs, and other ECM proteins via electrostatic interactions $[18,19]$. It was previously demonstrated that VEGF and PDGF-BB have different affinities to sulphated polymers [62]. Therefore, the higher density of sulfate groups of CNCs (sulfation density of $0.37 \pm 0.01 \mathrm{mmol} / \mathrm{g}$; supplementary information S3.1) may play a significant role on the different GFs release trends observed for these hydrogels. We hypothesize that CNCs can potentially mimic the sulfated glycosaminoglycans function. In a recent study, CNCs were shown to create anticoagulant surface coatings, mimicking the role of heparin [63]. Interestingly, this electrostatic interaction between CNCs and VEGF or PDGF-BB was further confirmed by Western Blotting, after GFs being extracted from the minced hydrogel pellets under denaturing conditions. Overall, the intensity of the bands varied with the incorporation of a-CNCs, becoming much less intense or almost nonexistent on CNCs-incorporating hydrogels. This may represent a simple way of adjusting the cell instructive cues of engineered 2D/3D constructs, according with the target applications.

Studies demonstrated the ability of PDGF and VEGF to upregulate the migration, and proliferation of hDPCs, the differentiation of odontoblasts, and to promote the angiogenesis activity [64]. In fact, to promote the recruitment of endogenous or transplanted cells and the revascularization of the root canal system, these GFs should interplay with cytokines in a temporal as spatial controlled manner. Therefore, the controlled release of GFs through the incorporation of CNCs is a real asset to the developed hydrogels.

The ability of hydrogels to support and control biochemical and cellular mechanisms is essential for TE applications. The hDPCs constitute an excellent stem cell source for dental tissue regeneration, owing to their high accessibility, proliferative ability, and clonogenic and multilineage differentiation potential [65-68]. Interestingly, the hDPCs encapsulated in HA hydrogels without CNCs incorporation, plain or PL-laden demonstrated higher cell cargo in almost all time points of culturing. Their soft characteristic and lower cross-linking density, might facilitate faster nutrient diffusion and matrix remodelling, thus promoting cellular activities such as spreading and migration, as previously reported on other studies $[69,70]$. The lower values for hydrogels with $0.25 \%$ and $0.5 \%$ CNCs content can be associated with a possible cytotoxic effect of the CNCs over the hDPCs. Some studies corroborate this theory suggesting that cytotoxic responses can occur at high CNCs concentrations, with significantly decreased cell viability above $200 \mu \mathrm{g} \mathrm{ml}^{-1}$ of CNCs [71], tissue damage, and inflammatory responses [72,73]. Remarkably, the adverse effects of CNCs seem to be overcome by the incorporation of PL into the hydrogels. Our findings show that incorporation of PL enhanced the proliferation and metabolic activity of encapsulated hDPCs, regulated by the growth factor milieu contained within the PL-laden scaffolds. The content in GFs allows PL to be used as a support for cellular processes such as chemotaxis [74], cell proliferation [75] and differentiation $[76,77]$. In addition, PL contains fibrinogen (precursor of fibrin), fibronectin and vitronectin which are important for ECM synthesis and for cells adhesion and migration [30,36]. Previous works have reported the positive effect of appropriate concentrations of supplemented PL in the proliferation and mineralized differentiation of human dental pulp stem cells, both in vitro and in vivo $[78,79]$. Thus, the developed hydrogels can be used as a cell delivery vehicle in TE strategies that also retains the nutritive functions of soluble PL, thereby enabling hDPCs survival, engraftment and delivery.

After having studied the release of chemotactic and proangiogenic GFs from PL-laden hydrogels and the ability of the hydrogels to support cell viability, we have studied the chemotactic and proangiogenic properties of the developed hydrogels. It has been shown that endothelial cells, either co-cultured with human mesenchymal stem cells (MSCs), perivascular lineages or alone, selfassembled to form microvascular networks $[1,4,80,81]$. This tendency becomes more pronounced when these cells are encapsulated in ECM-derived or chemically modified hydrogels following intrinsic homing mechanisms and cross-talk of cell and biomaterial [82]. In addition, the presence of GFs and signaling markers such as VEGF and PDGF can boost the mitogenic effect over cells, and the microvessel formation process $[64,82]$. In support of these findings, we tested PL gel's effect on hDPCs (which have been shown to contain MSCs populations [78]), invasion in a natural nutrition provision mimicking environment of the root canal system. It was quite evident that hDPCs in PL-loaded hydrogels had significantly greater invasion than in hydrogels without PL. Regarding the cocultures, PL-laden hydrogels also led to superior hDPCs sprouting when compared with HA/CNCs hydrogels, however, the HUVECs had little contribution within the sprouting cells. On the other hand, HUVECs seemed to enhance the hDPCs migration and formation of tubular-like structures. As hypothesized, PL incorporation influenced the migration of hDPCs; PL hydrogels contains a targeted growth factor milieu that induces migratory activity of hDPCs with minimal effect on sprouting of HUVECs. Indeed, the release of GFs from HA matrices has been demonstrated to have beneficial effects on cell viability and scaffold vascularization $[83,84]$. Similarly to the results that Robinson et al. [80] obtained in his work with MSCs/HUVECs incorporated into a PL hydrogel, when incorporated into the HA/CNCs hydrogels, PL encouraged hDPCs sprouting and initial formation of similar neovascular structures. The presence of in vitro cell-to-cell networks even without a very defined lumen (Fig. 6G) can be considered an early indicator of the formation of functionally integrated microvessels-like networks. Besides the influence of the PL incorporation, the low stiffness of the PL-loaded hydrogels may have contributed to the favorable cells invasion discovered here. These results are consistent with previous studies indicating that MSCs invasion is dependent on matrix stiffness with preferential growth in softer substrates [85-87]. In addition, the hydrogels microstructure also seems to condition the hDPCs migration. Open porous and interconnected networks are essential for cell nutrition, proliferation, infiltration and migration for tissue vascularization and new tissue ingrowth $[88,89]$. The migration peak evidenced in the chemotactic and proangiogenic assays coincides with the peak of hydrogels' porosity. Indeed, the preferential spreading observed on $0.25 \%$ $\mathrm{CNC} / \mathrm{PL}$ formulation can be ascribed to their higher pore size.

Finally, the ability of the injectable system to promote angiogenesis was also assessed in an ex vivo CAM assay. Several previous works have reported the pro-angiogenic potential of PL-based hydrogels [81], and the positive effect of conditioned medium from hDPCs $[90,91]$ in promoting the sprouting of endothelial cells, by releasing pro-angiogenic GFs (such as VEGF) that have a paracrine effect on the vascular cells. The combined effect of PL and hDPCs incorporation is evident in the present study by the promotion of the CAM infiltration within the PL-laden hydrogels containing 
$0.25 \mathrm{wt} \% \mathrm{CNCs}$, and the migration and arrangement of the hDPCs in the vicinity of new primordial microscopic blood vessels. Primary cultures isolated from dental pulp contain immature stem/progenitor cells together with fibroblasts, endothelial cells, and pericytes released from the pulp tissue [67]. Moreover, co-cultures demonstrate that DPCs participate in the formation of more stable blood vessels by promoting endothelial cells proliferation and migration covering HUVECs, acting as pericytes [92]. According to our results, besides expressing mesenchymal stem cell markers, primary isolated hDPCs were also positive for perivascular markers, namely $\alpha$-SMA, suggestive of their perivascular origin, as reported in previous studies [92,93]. In this sense, the incorporation of hDPCs in this novel injectable system might enhance the revascularization and new tissue ingrowth.

Overall, we suggest promising results of a novel HA-based hydrogel enriched with PL that has many of the desired characteristics needed for targeted vascular regeneration and tissue ingrowth of injured soft tissues with limited blood supply including favourable gel density, sustained gel integrity, a superior GFs retention and release profile, and the ability to promote cells' survival and migration. In this sense, endodontic regenerative therapies can take advantage of this injectable system for the recruitment of progenitor cells that survived apically in the preserved pulp tissue, and the revascularization of root canal system.

\section{Conclusions}

In this work, we detail the development of an injectable $\mathrm{HA} / \mathrm{CNCS} / \mathrm{PL}$ hydrogel, with desirable structural properties and biological activity. The stability, the porosity, and the dynamic viscoelastic behavior were changed by incorporating PL or CNCs, offering a significant degree of control over the hydrogel. The PL content significantly improved the viability of the encapsulated hDPCs, while augmented the hydrogels ability to recruit dental pulp-origin cells in vitro and promote cells sprouting in an ex vivo model, through the sustained release of chemotactic and pro-angiogenic GFs. These results, combined with the fact that these hydrogels can be easily injected into any desired defect and crosslinked in situ, clearly suggest the potential of this hydrogel in future developments for vascularized TE applications, such as in regenerative dentistry.

\section{Acknowledgements}

This work was supported by RECOGNIZE project (UTAP-ICDT/CTMBIO/0023/2014), project NORTE-01-0145-FEDER-000021. R.M.A. Domingues acknowledges FCT for SFRH/BPD/112459/2015. P.S. Babo acknowledges the project FOOD4CELLS (PTDC/CTM-BI O/4706/2014-POCI-01-0145-FEDER 016716) for his post-doc grant. The authors would like to thank Bárbara Mendes for the support in the precursors production, Ana Gonçalves for the supporting in the in vitro assays, Lucília Silva for HUVECs culturing, Ana Catarina Pinto for the cell tracking protocols used in the migration assays, and Maló Clinic, Porto, Dra Ana Ferro and Dr Bruno Queridinha for the donation of permanent teeth.

\section{Conflict of interest}

The authors have no conflicting financial interests.

\section{Data availability}

The raw/processed data required to reproduce these findings cannot be shared at this time as the data also forms part of an ongoing study.

\section{Appendix A. Supplementary data}

Supplementary data associated with this article can be found, in the online version, at https://doi.org/10.1016/j.actbio.2018.07.035.

\section{References}

[1] M.P. Cuchiara, D.J. Gould, M.L. McHale, M.E. Dickinson, J.L. West, Integration of self-assembled microvascular networks with microfabricated PEG-based hydrogels, Adv. Funct. Mater. 22 (2010) 4511-4518.

[2] J.S. Miller, K.R. Stevens, M.T. Yang, B.M. Baker, D.H.T. Nguyen, D.M. Cohen, E Toro, A.A. Chen, P.A. Galie, X. Yu, R. Chaturvedi, S.N. Bhatia, C.S. Chen, Rapid casting of patterned vascular networks for perfusable engineered threedimensional tissues, Nat. Mater. 11 (2012) 768-774.

[3] T.M. Botero, J.E. Nör, Tissue engineering strategies for endodontic regeneration, Stem Cell Biol.Tissue Eng. Dent. Sci. (2015) 419-430.

[4] H. Bae, A.S. Puranik, R. Gauvin, F. Edalat, B. Carrillo-, N.A. Peppas, A. Khademhosseini, Building vascular networks, Sci. Transl. Med. 4 (2012) 1-12.

[5] H.-H. Sun, T. Jin, Y. Qing, F.-M. Chen, Biological approaches toward dental pulp regeneration by tissue engineering, J. Tissue Eng. Regen. Med. 5 (2011) 1-16.

[6] J.Y. Kim, X. Xin, E.K. Moioli, J. Chung, C.H. Lee, M. Chen, S.Y. Fu, P.D. Koch, J.J. Mao, Regeneration of dental-pulp-like tissue by chemotaxis-induced cell homing, Tissue Eng. Part A 16 (2010) 3023-3031.

[7] P. Amrollahi, B. Shah, A. Seifi, L. Tayebi, Recent advancements in regenerative dentistry: a review, Mater. Sci. Eng. C. 69 (2016) 1383-1390.

[8] N.B. Graham, Hydrogels: their future, Part I, Med. Device Technol. 9 (1998) 1822.

[9] B. Jeong, Y.H. Bae, D.S. Lee, S.W. Kim, Biodegradable block copolymers as injectable drug-delivery systems, Nature 388 (1997) 860-862.

[10] A. Chenite, C. Chaput, D. Wang, C. Combes, M.D. Buschmann, C.D. Hoemann, J. C. Leroux, B.L. Atkinson, F. Binette, A. Selmani, Novel injectable neutral solutions of chitosan form biodegradable gels in situ, Biomaterials 21 (2000) 2155-2161.

[11] J.S. Temenoff, A.G. Mikos, Injectable biodegradable materials for orthopedic tissue engineering, Biomaterials 21 (2000) 2405-2412.

[12] R. Zhang, M. Xue, J. Yang, T. Tan, A novel injectable and in situ crosslinked hydrogel based on hyaluronic acid and $\alpha, \beta$-polyaspartylhydrazide, J. Appl. Polym. Sci. 125 (2012) 1116-1126.

[13] R.M.A. Domingues, M. Silva, P. Gershovich, S. Betta, P. Babo, A. Motta, R.L. Reis, M.E. Gomes, Development of injectable hyaluronic acid/cellulose nanocrystals bionanocomposite hydrogels for tissue engineering applications, Bioconjug. Chem. 26 (2015) 1571-1581.

[14] B.P. Toole, Hyaluronan: from extracellular glue to pericellular cue, Nat. Rev. Cancer. 4 (2004) 528-539.

[15] L. Ferroni, C. Gardin, S. Sivolella, G. Brunello, M. Berengo, A. Piattelli, E. Bressan, B. Zavan, A hyaluronan-based scaffold for the in vitro construction of dental pulp-like tissue, Int. J. Mol. Sci. 16 (2015) 4666-4681.

[16] C.R. Silva, M. Gomez-Florit, P.S. Babo, R.L. Reis, M.E. Gomes, 3D Functional scaffolds for dental tissue engineering, in: Y. Deng, J. Kuiper (Eds.), Funct. 3D Tissue Eng. Scaffolds - Mater. Technol. Appl., 1st ed., Elsevier, 2017, pp. 423450.

[17] X. Yang, E. Bakaic, T. Hoare, E.D. Cranston, Injectable polysaccharide hydrogels reinforced with cellulose nanocrystals: morphology, Rheol. Degrad. Cytot. Biomacromol. 14 (2013) 4447-4455.

[18] J. Salbach, T.D. Rachner, M. Rauner, U. Hempel, U. Anderegg, S. Franz, J.C. Simon, L.C. Hofbauer, Regenerative potential of glycosaminoglycans for skin and bone, J. Mol. Med. 90 (2012) 625-635.

[19] R.A. Hortensius, B.A.C. Harley, The use of bioinspired alterations in the glycosaminoglycan content of collagen-GAG scaffolds to regulate cell activity, Biomaterials 34 (2013) 7645-7652.

[20] L.G. Chase, U. Lakshmipathy, L.A. Solchaga, M.S. Rao, M.C. Vemuri, A novel serum-free medium for the expansion of human mesenchymal stem cells, Stem Cell Res. Ther. 1 (2010) 8.

[21] D. Bondeson, A. Mathew, K. Oksman, Optimization of the isolation of nanocrystals from microcrystalline cellulose by acid hydrolysis, Cellulose 13 (2006) 171-180.

[22] R. Dash, M. Foston, A.J. Ragauskas, Improving the mechanical and thermal properties of gelatin hydrogels cross-linked by cellulose nanowhiskers, Carbohydr. Polym. 91 (2013) 638-645.

[23] U.J. Kim, S. Kuga, M. Wada, T. Okano, T. Kondo, Periodate oxidation of crystalline cellulose, Biomacromolecules 1 (2000) 488-492.

[24] K.P. Vercruysse, D.M. Marecak, J.F. Marecek, G.D. Prestwich, Synthesis and in vitro degradation of new polyvalent hydrazide cross-linked hydrogels of hyaluronic acid, Bioconjugate Chem. 8 (1997) 686-694.

[25] Y. Yeo, C.B. Highley, E. Bellas, T. Ito, R. Marini, R. Langer, D.S. Kohane, In situ cross-linkable hyaluronic acid hydrogels prevent post-operative abdominal adhesions in a rabbit model, Biomaterials. 27 (2006) 4698-4705.

[26] J. Dahlmann, A. Krause, L. Möller, G. Kensah, M. Möwes, A. Diekmann, U. Martin, A. Kirschning, I. Gruh, G. Dräger, Fully defined in situ cross-linkable alginate and hyaluronic acid hydrogels for myocardial tissue engineering, Biomaterials. 34 (2013) 940-951.

[27] H. Tan, C.R. Chu, K.A. Payne, K.G. Marra, Injectable in situ forming biodegradable chitosan - hyaluronic acid based hydrogels for cartilage tissue engineering, Biomaterials. 30 (2009) 2499-2506. 
[28] V.E. Santo, M.E. Gomes, J.F. Mano, R.L. Reis, Chitosan - chondroitin sulphate nanoparticles for controlled delivery of platelet lysates in bone regenerative medicine, J. Tissue Eng. Regen. Med. 6 (2012) 47-59.

[29] L.F.D. Almeida, P.S. Babo, C.R. Silva, M. Rodrigues, J. Hebling, R.L. Reis, M.E. Gomes, Hyaluronic acid hydrogels incorporating platelet lysate enhance human pulp cell proliferation and differentiation, J. Mater. Sci. Mater (2018).

[30] P. Babo, V.E. Santo, A.R.C. Duarte, C. Correia, M.H.G. Costa, J.F. Mano, R.L. Reis, M.E. Gomes, Platelet lysate membranes as new autologous templates for tissue engineering applications, Inflamm. Regen. 34 (2014) 33-44.

[31] J. Silva-Correia, V. Miranda-Gonçalves, A.J. Salgado, N. Sousa, J.M. Oliveira, R.M. Reis, R.L. Reis, Angiogenic potential of Gellan-Gum-based hydrogels for application in nucleus pulposus regeneration: in vivo study, Tissue Eng. Part A 18 (2012) 1203-1212.

[32] M.N. Collins, C. Birkinshaw, Hyaluronic acid based scaffolds for tissue engineering - a review, Carbohydr. Polym. 92 (2013) 1262-1279.

[33] J.A. Burdick, C. Chung, X. Jia, M.A. Randolph, R. Langer, Controlled degradation and mechanical behavior of photopolymerized hyaluronic acid networks, Biomacromolecules 6 (2005) 386-391.

[34] E. Anitua, I. Andia, B. Ardanza, P. Nurden, A.T. Nurden, Autologous platelets as a source of proteins for healing and tissue regeneration, Thromb. Haemost. 91 (2004) 4-15.

[35] X. Tan, H. Ju, W. Yan, H. Jiang, J. Su, H. Dong, L. Wang, D. Zou, Autologous platelet lysate local injections for the treatment of refractory lateral epicondylitis, J. Orthop. Surg. Res. 11 (2016) 17.

[36] N. Fekete, M. Gadelorge, D. Furst, C. Maurer, J. Dausend, S. Fleury-Cappellesso, V. Mailander, R. Lotfi, A. Ignatius, L. Sensebé, P. Bourin, H. Schrezenmeier, M.T. Rojewski, Platelet lysate from whole blood-derived pooled platelet concentrates and apheresis-derived platelet concentrates for the isolation and expansion of human bone marrow mesenchymal stromal cells: production process, content and identification of active comp, Cytotherapy 14 (2012) 540-554.

[37] P.H. Warnke, A. Humpe, D. Strunk, S. Stephens, F. Warnke, J. Wiltfang, K. Schallmoser, M. Alamein, R. Bourke, P. Heiner, Q. Liu, A clinically-feasible protocol for using human platelet lysate and mesenchymal stem cells in regenerative therapies, J. Cranio-Maxillofacial. Surg. 41 (2013) 153-161.

[38] V. Govindasamy, V.S. Ronald, A.N.B. Abdullah, K.R. Ganesan Nathan, Z.A.C.A. Aziz, M. Abdullah, R.B. Zain, N.H.A. Kasim, S. Musa, R.R. Bhonde, Human platelet lysate permits scale-up of dental pulp stromal cells for clinical applications, Cytotherapy 13 (2011) 1221-1233.

[39] R. Crespo-Diaz, A. Behfar, G.W. Butler, D.J. Padley, M.G. Sarr, J. Bartunek, A.B. Dietz, A. Terzic, Platelet lysate consisting of a natural repair proteome supports human mesenchymal stem cell proliferation and chromosomal stability, Cell Transplant. 20 (2011) 797-811.

[40] G. Weibrich, W.K.G. Kleis, G. Hafner, W.E. Hitzler, Growth factor levels in platelet-rich plasma and correlations with donor age, sex, and platelet count, J. Cranio-Maxillofacial. Surg. 30 (2002) 97-102.

[41] R.P. Russell, J. Apostolakos, T. Hirose, M.P. Cote, A.D. Mazzocca, Variability of platelet-rich plasma preparations, Sports Med. Arthrosc. 21 (2013) 186-190.

[42] B.L. Eppley, J.E. Woodell, J. Higgins, Platelet quantification and growth factor analysis from platelet-rich plasma: implications for wound healing, Plast. Reconstr. Surg. 114 (2004) 1502-1508.

[43] I.B. Copland, M.A. Garcia, E.K. Waller, J.D. Roback, J. Galipeau, The effect of platelet lysate fibrinogen on the functionality of MSCs in immunotherapy, Biomaterials 34 (2013) 7840-7850.

[44] D.A. Ossipov, S. Piskounova, O.P. Varghese, J. Hilborn, Functionalization of hyaluronic acid with chemoselective groups via a disulfide-based protection strategy for in situ formation of mechanically stable hydrogels, Biomacromolecules 11 (2010) 2247-2254.

[45] T. Ito, Y. Yeo, C.B. Highley, E. Bellas, C.A. Benitez, D.S. Kohane, The prevention of peritoneal adhesions by in situ cross-linking hydrogels of hyaluronic acid and cellulose derivatives, Biomaterials 28 (2007) 975-983.

[46] D.E. Ingber, Cellular mechanotransduction: putting all the pieces together again, FASEB J. 20 (2006) 811-827.

[47] W.H. Guo, M.T. Frey, N.A. Burnham, Y.L. Wang, Substrate rigidity regulates the formation and maintenance of tissues, Biophys. J. 90 (2006) 2213-2220.

[48] O. Collin, P. Tracqui, A. Stephanou, Y. Usson, J. Clément-Lacroix, E. Planus, Spatiotemporal dynamics of actin-rich adhesion microdomains: influence of substrate flexibility, J. Cell Sci. 119 (2006) 1914-1925.

[49] J.D. Mih, A. Marinkovic, F. Liu, A.S. Sharif, D.J. Tschumperlin, Matrix stiffness reverses the effect of actomyosin tension on cell proliferation, J. Cell Sci. 125 (2012) 5974-5983.

[50] P.C. Georges, P.A. Janmey, Cell type-specific response to growth on soft materials, J. Appl. Physiol. 98 (2005) 1547-1553.

[51] A.J. Engler, M.A. Griffin, S. Sen, C.G. Bönnemann, H.L. Sweeney, D.E. Discher, Myotubes differentiate optimally on substrates with tissue-like stiffness: Pathological implications for soft or stiff microenvironments, J. Cell Biol. 166 (2004) 877-887.

[52] S.L. Thibeault, S.A. Klemuk, X. Chen, B.H.Q. Johnson, In vivo engineering of the vocal fold ECM with injectable HA hydrogels - late effects on tissue repair and biomechanics in a rabbit model, J. Voice. 25 (2011) 249-253.

[53] P.A. Band, Hyaluronan derivatives: Chemistry and clinical applications, in: T. Laurent (Ed.), Chem. Biol. Med. Appl. Hyaluronan Its Deriv., Portland Press Ltd., London, 1998, pp. 33-42.

[54] M. Kucur, B. Karadag, F.K. Isman, Y. Ataev, D. Duman, N. Karadag, Z. Ongen, V.A. Vural, Plasma hyaluronidase activity as an indicator of atherosclerosis in patients with coronary artery disease, Bratislava Med. J. 110 (2009) 21-26.
[55] J.-S. Tung, G.E. Mark, G.F. Hollis, A microplate assay for hyaluronidase and hyaluronidase inhibitors, Anal. Biochem. 223 (1994) 149-152.

[56] F.-M. Chen, Y. Jin, Periodontal tissue engineering and regeneration: current approaches and expanding opportunities, Tissue Eng. Part B. Rev. 16 (2010) 219-255.

[57] C. Zhou, Q. Wu, Y. Yue, Q. Zhang, Application of rod-shaped cellulose nanocrystals in polyacrylamide hydrogels, J. Colloid Interface Sci. 353 (2011) 116-123.

[58] P.S. Babo, P.P. Carvalho, V.E. Santo, S. Faria, M.E. Gomes, R.L. Reis, Assessment of bone healing ability of calcium phosphate cements loaded with platelet lysate in rat calvarial defects, J. Biomater. Appl. 31 (2016) 637-649.

[59] K. Hayashi, F. Okamoto, S. Hoshi, T. Katashima, D.C. Zujur, X. Li, M. Shibayama, E.P. Gilbert, U. Il Chung, S. Ohba, T. Oshika, T. Sakai, Fast-forming hydrogel with ultralow polymeric content as an artificial vitreous body, Nat, Biomed. Eng. 1 (2017) 1-7.

[60] E. Larrañeta, M. Henry, N.J. Irwin, J. Trotter, A.A. Perminova, R.F. Donnelly, Synthesis and characterization of hyaluronic acid hydrogels crosslinked using a solvent-free process for potential biomedical applications, Carbohydr. Polym. 181 (2018) 1194-1205.

[61] S.J. Kim, C.K. Lee, Y.M. Lee, I.Y. Kim, S.I. Kim, Electrical/pH-sensitive swelling behavior of polyelectrolyte hydrogels prepared with hyaluronic acid-poly (vinyl alcohol) interpenetrating polymer networks, React. Funct. Polym. 55 (2003) 291-298.

[62] S.M. Oliveira, V.E. Santo, M.E. Gomes, R.L. Reis, J.F. Mano, Layer-by-layer assembled cell instructive nanocoatings containing platelet lysate, Biomaterials 48 (2015) 56-65.

[63] H.M.A. Ehmann, T. Mohan, M. Koshanskaya, S. Scheicher, D. Breitwieser, V Ribitsch, K. Stana-Kleinschek, S. Spirk, Design of anticoagulant surfaces based on cellulose nanocrystals, Chem. Commun. 50 (2014) 13070-13072.

[64] M. Zhang, F. Jiang, X. Zhang, S. Wang, Y. Jin, W. Zhang, X. Jiang, The effects of platelet-derived growth factor-BB on human dental pulp stem cells mediated dentin-pulp complex regeneration, Stem Cells Transl. Med. 6 (2017) 2126 2134

[65] I. Thesleff, Epithelial-mesenchymal signalling regulating tooth morphogenesis J. Cell Sci. 116 (2003) 1647-1648.

[66] J. Pispa, I. Thesleff, Mechanisms of ectodermal organogenesis, Dev. Biol. 262 (2003) 195-205.

[67] S. Gronthos, M. Mankani, J. Brahim, P.G. Robey, S. Shi, Postnatal human dental pulp stem cells (DPSCs) in vitro and in vivo, Proc. Natl. Acad. Sci. 97 (2000) $13625-13630$

[68] S. Gronthos, J. Brahim, W. Li, L.W.W. Fisher, N. Cherman, A. Boyde, P. Denbesten, P.G. Robey, S. Shi, Stem cell properties of human dental pulp stem cells, J. Dent. Res. 81 (2002) 531-535.

[69] Y. Lei, S. Gojgini, J. Lam, T. Segura, The spreading, migration and proliferation of mouse mesenchymal stem cells cultured inside hyaluronic acid hydrogels, Biomaterials. 32 (2011) 39-47.

[70] H.J. Lee, A. Sen, S. Bae, J.S. Lee, K. Webb, Poly(ethylene glycol) diacrylate/ hyaluronic acid semi-interpenetrating network compositions for 3-D cell spreading and migration, Acta Biomater. 14 (2015) 43-52.

[71] M.M. Pereira, N.R.B. Raposo, R. Brayner, E.M. Teixeira, V. Oliveira, C.C.R Quintão, L.S.A. Camargo, L.H.C. Mattoso, H.M. Brandão, Cytotoxicity and expression of genes involved in the cellular stress response and apoptosis in mammalian fibroblast exposed to cotton cellulose nanofibers, Nanotechnology 24 (2013) 75103.

[72] M.J.D. Clift, E.J. Foster, D. Vanhecke, D. Studer, P. Wick, P. Gehr, B. Rothenrutishauser, C. Weder, Investigating the interaction of cellulose nanofibers derived from cotton with a sophisticated 3D human lung cell coculture, Biomacromolecules 12 (2011) 3666-3673.

[73] N. Yanamala, M.T. Farcas, M.K. Hatfield, E.R. Kisin, V.E. Kagan, C.L. Geraci, A.A. Shvedova, In vivo evaluation of the pulmonary toxicity of cellulose nanocrystals: a renewable and sustainable nanomaterial of the future, ACS Sustain. Chem. Eng. 2 (2014) 1691-1698.

[74] M.C. Phipps, Y. Xu, S.L. Bellis, Delivery of platelet-derived growth factor as a chemotactic factor for mesenchymal stem cells by bone-mimetic electrospun scaffolds, PLoS One 7 (2012) 1-9.

[75] K. Schallmoser, C. Bartmann, E. Rohde, A. Reinisch, K. Kashofer, Human platelet lysate can replace fetal bovine serum for clinical-scale expansion of functional mesenchymal stromal cells, Transfusion 47 (2007) 1436-1446.

[76] M. Matsui, Y. Tabata, Enhanced angiogenesis by multiple release of plateletrich plasma contents and basic fibroblast growth factor from gelatin hydrogels, Acta Biomater. 8 (2012) 1792-1801.

[77] J. Kurita, M. Miyamoto, Y. Ishii, J. Aoyama, G. Takagi, Z. Naito, Y. Tabata, M Ochi, K. Shimizu, Enhanced vascularization by controlled release of plateletrich plasma impregnated in biodegradable gelatin hydrogel, Ann Thorac Surg. 92 (2011) 837-844.

[78] B. Chen, H. Sun, H. Wang, H. Kong, F. Chen, Q. Yu, The effects of human platelet lysate on dental pulp stem cells derived from impacted human third molars, Biomaterials 33 (2012) 5023-5035.

[79] P. Marrazzo, F. Paduano, F. Palmieri, M. Marrelli, M. Tatullo, Highly efficient in vitro reparative behaviour of dental pulp stem cells cultured with standardised platelet lysate supplementation, Stem Cells Int. 2016 (2016) 116.

[80] S.T. Robinson, A.M. Douglas, T. Chadid, K. Kuo, A. Rajabalan, H. Li, I.B. Copland, T.H. Barker, J. Galipeau, L.P. Brewster, A novel platelet lysate hydrogel for endothelial cell and mesenchymal stem cell-directed neovascularization, Acta Biomater. 36 (2016) 86-98. 
81] T.M. Fortunato, C. Beltrami, C. Emanueli, P.A. De Bank, G. Pula, Platelet lysate gel and endothelial progenitors stimulate microvascular network formation in vitro: tissue engineering implications, Sci. Rep. 6 (2016) 25326.

[82] N. Annabi, A. Tamayol, J.A. Uquillas, M. Akbari, L.E. Bertassoni, C. Cha, G. CamciUnal, M.R. Dokmeci, N.A. Peppas, A. Khademhosseini, 25th anniversary article: rational design and applications of hydrogels in regenerative medicine, Adv. Mater. 26 (2014) 85-124.

[83] R.A. Peattie, E.R. Rieke, E.M. Hewett, R.J. Fisher, X.Z. Shu, G.D. Prestwich, Dua growth factor-induced angiogenesis in vivo using hyaluronan hydrogel implants, Biomaterials 27 (2006) 1868-1875.

[84] D.B. Pike, S. Cai, K.R. Pomraning, M.A. Firpo, R.J. Fisher, X.Z. Shu, G.D. Prestwich, R.A. Peattie, Heparin-regulated release of growth factors in vitro and angiogenic response in vivo to implanted hyaluronan hydrogels containing VEGF and bFGF, Biomaterials 27 (2006) 5242-5251.

[85] A.J. Hanson, M.T. Quinn, Effect of fibrin sealant composition on human neutrophil chemotaxis, J. Biomed. Mater. Res. 61 (2002) 474-481.

[86] W. Ho, B. Tawil, J.C.Y. Dunn, B.M. Wu, The behavior of human mesenchyma stem cells in 3D fibrin clots: dependence on fibrinogen concentration and clot structure, Tissue Eng. 12 (2006) 1587-1595.

[87] A. Stolzing, H. Colley, A. Scutt, Effect of age and diabetes on the response of mesenchymal progenitor cells to fibrin matrices, Int. J. Biomater. 2011 (2011) $1-9$.
[88] F. Causa, P.A. Netti, L. Ambrosio, A multi-functional scaffold for tissue regeneration: the need to engineer a tissue analogue, Biomaterials 28 (2007) 5093-5099.

[89] N. Annabi, J.W. Nichol, X. Zhong, C. Ji, S. Koshy, A. Khademhosseini, F. Dehghani, Controlling the porosity and microarchitecture of hydrogels for tissue engineering, Tissue Eng. Part B Rev. 16 (2010) 371-383.

[90] W.L. Dissanayaka, K.M. Hargreaves, L. Jin, L.P. Samaranayake, C. Zhang, The interplay of dental pulp stem cells and endothelial cells in an injectable peptide hydrogel on angiogenesis and pulp regeneration in vivo, Tissue Eng. Part A 21 (2015) 550-563.

[91] C. Gorin, G.Y. Rochefort, R. Bascetin, H. Ying, J. Lesieur, J. Sadoine, N. Beckouche, S. Berndt, A. Novais, M. Lesage, B. Hosten, L. Vercellino, P. Merlet, D. LeDenmat, C. Marchiol, D. Letourneur, A. Nicoletti, S.O. Vital, A. Poliard, B. Salmon, L. Muller, C. Chaussain, S. Germain, Priming dental pulp stem cells with fibroblast growth factor- 2 increases angiogenesis of implanted tissueengineered constructs through hepatocyte growth factor and vascular endothelial growth factor secretion, Stem Cells Transl. Med. 5 (2016) 392-404.

[92] W.L. Dissanayaka, X. Zhan, C. Zhang, K.M. Hargreaves, L. Jin, E.H.Y. Tong, Coculture of dental pulp stem cells with endothelial cells enhances osteo-/ odontogenic and angiogenic potential in vitro, JOE 38 (2012) 454-463.

[93] H. Nam, G.H. Kim, Y.K. Bae, D.E. Jeong, K.M. Joo, K. Lee, S.H. Lee, Angiogenic capacity of dental pulp stem cell regulated by SDF- $1 \alpha$-CXCR 4 axis, Stem Cells Int. (2017) 1-10. 